\title{
Suppressor of cytokine signaling 6 (SOCS6) promotes mitochondrial fission via regulating DRP1 translocation
}

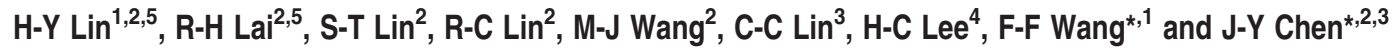

Mitochondria are highly motile organelles that constantly undergo fission and fusion. Impairment of mitochondrial dynamics is associated with mitochondrial dysfunction and is frequently linked to the pathogenesis of neurodegenerative diseases and cancer. We have previously shown that biallelic inactivation of the suppressor of cytokine signaling 6 (SOCS6) gene is a frequent event in human gastric cancer. In this study, we recapitulated the event of SOCS6 loss using a Lentivirus-based knockdown approach, and demonstrated the linkage between SOCS6 depletion and the suppression of programmed cell death. SOCS6 promotes intrinsic apoptosis, with increased Bax conformational change, mitochondrial targeting, and oligomerization. Most importantly, SOCS6 is targeted to mitochondria and induces mitochondrial fragmentation mediated through an increase in DRP1 fission activity. Here, we show that SOCS6 forms complex with DRP1 and the mitochondrial phosphatase PGAM5, attenuates DRP1 phosphorylation, and promotes DRP1 mitochondrial translocation. Based on mutation analyses, SOCS6-mediated apoptosis is tightly coupled to its ability to induce mitochondrial fission. This study demonstrates an important role for SOCS6 in modulating mitochondrial dynamics and apoptosis.

Cell Death and Differentiation (2013) 20, 139-153; doi:10.1038/cdd.2012.106; published online 7 September 2012

Mitochondria are essential organelles in cellular metabolism and respiration. In addition, mitochondria also act as cell death executors to initiate apoptosis by releasing cytochrome $c$ and other proapoptotic factors. ${ }^{1,2}$ Mitochondria are highly dynamic that undergo continuous fission and fusion processes, ${ }^{3-5}$ which are important for maintaining mitochondrial morphology, distribution, and functions. Unbalanced mitochondrial dynamics has been implicated in a number of human pathologies, including cancer ${ }^{6}$ and neurodegenerative diseases. $^{7}$

Mitochondrial fission and fusion machineries are governed by the balanced yet opposing actions of a number of large GTPase dynamin family proteins. ${ }^{8}$ In mammalian cells, the mitochondrial fusion machinery is regulated by mitofusins 1 and $2(\mathrm{MFN} 1 / 2)^{9}$ and optic atrophy 1 (OPA1), ${ }^{10}$ whereas the fission process is driven by dynamin-related protein 1 $(\mathrm{DRP} 1)^{11}$ and its mitochondrial anchor proteins mitochondrial fission factor (Mff) ${ }^{12}$ and fission 1 (Fis 1$) .^{13}$

During apoptosis, mitochondria undergo a significant morphological change from a filamentous network to punctate fragments. By binding to its anchoring proteins on the mitochondrial outer membrane, DRP1 is recruited from the cytoplasm to mitochondria, and assembled into spiral-shaped multimeric complex at the scission sites, where it constricts the organelles. ${ }^{11,14}$ The activity of DRP1 is regulated by posttranslational modifications. ${ }^{15}$ Phosphorylation of DRP1-Ser ${ }^{637}$ by cyclic AMP-dependent kinase (PKA) inhibited DRP1 translocation to the mitochondria, ${ }^{16}$ whereas calcineurin-dependent dephosphorylation of this residue increased its recruitment to mitochondria. ${ }^{17}$ In addition, the mitochondrial outer membrane protein phosphoglycerate mutase 5 (PGAM5), which has no apparent phosphoglycerate mutase activity but displays phosphoserine/threonine phosphatase activity, has recently been shown to play an important role in the initiation of necrosis by dephosphorylating DRP1Ser $^{637}$ and promoting DRP1 mitochondrial translocation. ${ }^{18}$ Mounting evidence has suggested that mitochondrial fission plays an active role in apoptosis. DRP1-induced excessive mitochondrial fission caused ectopic programmed cell death, ${ }^{19}$ and inhibition of DRP1 activity by a dominantnegative mutant $^{20}$ or a chemical inhibitor ${ }^{21}$ delayed programmed cell death. Furthermore, inhibition of mitochondrial

\footnotetext{
${ }^{1}$ Institute of Biochemistry and Molecular Biology, National Yang-Ming University, Taipei, Taiwan; ${ }^{2}$ Institute of Biomedical Sciences, Academia Sinica, Taipei, Taiwan; ${ }^{3}$ Department of Life Sciences and Institute of Genome Sciences, National Yang-Ming University, Taipei, Taiwan and ${ }^{4}$ Institute of Pharmacology, National Yang-Ming University, Taipei, Taiwan

*Corresponding author: J-Y Chen, Institute of Biomedical Sciences, Academia Sinica, 128 Academia Road, Section 2, Taipei 115, Taiwan. Tel: +886 2 2789 9046; Fax: +886 22785 8594; E-mail: bmchen@ibms.sinica.edu.tw

or F-F Wang, Institute of Biochemistry and Molecular Biology, National Yang-Ming University, 155 Li-Nong Street, Section 2, Shih-Pai, Taipei 112, Taiwan.

Tel: +886 22826 7126; Fax: +886 22826 4843; E-mail: ffwang@ym.edu.tw

${ }^{5}$ These authors contributed equally to this work.

Keywords: apoptosis; DRP1; mitochondrial dynamic; PGAM5; SOCS6

Abbreviations: Bcl-2, B-cell lymphoma 2; Drp1, dynamin-related protein 1; ECS, Elongin BC-Cul2/Cul5-SOCS box protein; EYFP, enhanced yellow fluorescent proteins; FRAP, fluorescence recovery after photobleaching; GPI, glycosylphosphatidylinositol; HA, hemagglutinin; MFN, mitofusin; OMM, outer mitochondrial membrane; PGAM5, phosphoglycerate mutase 5; SOCS6, suppressor of cytokine signaling 6; STS, staurosporin; UV, ultraviolet

Received 23.1.12; revised 09.7.12; accepted 31.7.12; Edited by L Scorrano; published online 07.9.12
} 
translocation of DRP1 by a calcineurin antagonist delayed intrinsic apoptosis pathway. ${ }^{22}$

We previously showed that loss of suppressor of cytokine signaling 6 (SOCS6) is a frequent event in human gastric cancer, and that ectopic expression of SOCS6 promotes cell death. ${ }^{23}$ SOCS6 belongs to the family of SOCS proteins, several of which have been characterized as negative feedback regulators for cytokine receptor signaling. ${ }^{24}$ However, SOCS6 does not interact with cytokine signaling intermediate molecules or inhibit cytokine receptor signaling. ${ }^{24,25}$ To further elucidate the suppressive function of SOCS6, in this study, we knocked down the expression of SOCS6 and showed that loss of SOCS6 promoted cell survival upon apoptotic insults. Our data demonstrate that SOCS6 encodes a novel mitochondrial targeting protein involved in the regulation of mitochondrial dynamics and functions through attenuating DRP1 phosphorylation and promoting its translocation to mitochondria.

\section{Results}

Loss of SOCS6 confers cells resistance to the intrinsic apoptosis pathway. To examine how the loss of SOCS6 may contribute to cell transformation, we examined the effect of SOCS6 knockdown (KD) on ultraviolet (UV)-induced apoptosis in HeLa cells. The introduction of two independent SOCS6-shRNAs led to varying degrees of SOCS6 mRNA and protein KD compared with the control shRNA that targeted luciferase mRNA (Figure 1a). Based on the KD effect, HeLa cell clone stably harboring SOCS6-shRNA1 was established for further examination. Reduction of endogenous SOCS6 yielded cells more resistant to UV-induced apoptosis, demonstrated by the decreased numbers of annexin-V-positive cells (Figure $1 \mathrm{~b}$ ), reduced levels of PARP and caspase 3 cleavage (Figure 1c), lowered sub-G1 populations (Figure 1d), and delayed cytochrome $c$ release (Figure 1e). In contrast, cells expressing ectopic SOCS6 were more sensitive to UV-induced apoptosis (Figure 1f), but not tumor necrosis factor- $\alpha$ (TNF- $\alpha$ )-induced apoptosis (data not shown). These data suggest that ectopic expression of SOCS6 promotes apoptosis through an intrinsic pathway and that KD of SOCS6 inhibits this process.
Upon initiation of intrinsic apoptosis, Bax undergoes a conformational change allowing stepwise structural reorganization for mitochondrial targeting and homo-oligomerization. ${ }^{26}$ Ectopic expression of SOCS6 in HeLa cells promoted a Bax conformational change, leading to the exposure of the $\mathrm{N}$-terminal epitope, in a dose-dependent manner (Figure 1g). Consequently, increased localization and accumulation of Bax in mitochondria were observed in SOCS6-expressing cells (Figure 1h). We also showed that increasing concentrations of staurosporin (STS) induced Bax conformational change and dimer formation in a dose-dependent manner, and that ectopic expression of SOCS6 further enhanced these processes (Figures 1i and j). In contrast, KD of SOCS6 significantly inhibited STS-induced Bax conformational changes and dimer formation (data not shown). These data suggest that ectopic expression of SOCS6 promotes Bax activation and that KD of SOCS6 attenuates STS-induced Bax activation.

SOCS6 encodes a mitochondrial targeting protein. To investigate the function of SOCS6, we examined the intracellular localization of SOCS6. As shown in Figure 2a, the ectopically expressed human influenza hemagglutinin (HA)-tagged SOCS6 displayed a punctuate distribution and colocalized largely with the mitochondria-targeted enhanced yellow fluorescent proteins (EYFPs) in $>70 \%$ of the transfected cells. Endogenous SOCS6 was expressed at relatively low levels across various cell lines. Nevertheless, a major proportion of the endogenous SOCS6 was localized to mitochondria (Figure 2b). By differential centrifugation, we show that HA-SOCS6 occurred mainly in the fractions enriched for heavy mitochondria (Figure 2c). By subcellular fractionation, the majority of ectopic as well as endogenous SOCS6 was recovered from the mitochondrial fraction (Figure 2d). The mitochondrial fraction was subjected to trypsin digestion in isotonic and hypotonic buffers. SOCS6 was sensitive to trypsin digestion in the isotonic buffer as was the outer mitochondrial membrane (OMM) protein B-cell lymphoma 2 (Bcl-2), suggesting that SOCS6 is located in the OMM (Figure 2e). By resuspending the isolated mitochondria in $0.1 \mathrm{M}$ sodium carbonate $(\mathrm{pH} 11.5)$ or $0.5 \mathrm{M}$ sodium chloride, we further show that SOCS6 was

Figure 1 Loss of SOCS6 confers cells resistance to apoptotic insults. (a) The expressions of SOCS6 in HeLa cells stably harboring shRNAs targeting at luciferase (controlKD) and SOCS6 (SOCS6-KD) were assessed by RT-PCR and western blot analyses. Two individual shRNAs targeting SOCS6 were employed. (b-e) HeLa cells stably harboring shRNA directed against luciferase and SOCS6 were irradiated with UV at $90 \mathrm{~J} / \mathrm{m}^{2}$. After incubation for the designated times, cells were harvested for apoptosis assays. Data derived from three separate experiments are presented as the means \pm S.E.M. (b) Annexin $V$ staining of phosphatidylserine exposed on the cell surface was measured by flow cytometric analysis. (c) Total cell lysates were prepared for western blot analysis of the designated proteins. (d) Cells were stained with propidium iodide and subjected to FACS analysis of sub-G1 population. (e) Cells were labeled with MitoTracker and immunostained using an anti-cytochrome $c$ antibody followed by labeling with Alexa 488 and counterstaining with DAPI. Representative immunofluorescence images are shown. Cells that released cytochrome $c$, as indicated by arrows, were scored at the designated time points and presented as percentage of total cells in a bar graph. Scale bar: $10 \mu \mathrm{m}$. (f-j) Ectopic expression of SOCS6 promotes apoptosis and Bax activation. (f) AD293 cells were transfected with pcDNA3-SOCS6 (SOCS6) or the control vector (mock), UV irradiated, and subjected to FACS analysis of sub-G1 population at the designated times. Data derived from three separate experiments are presented as the means \pm S.E.M. (g) HeLa cells were transfected with a control vector or increasing amounts of pcDNA3-SOCS6. Cells were lysed in CHAPS buffer and immunoprecipitated using an anti-Bax monoclonal antibody, 6A7. Immunoprecipitates were subjected to western blotting using an anti-Bax polyclonal antibody (N-20). (h) Mock- and HA-SOCS6-transfected HeLa cells were treated with or without UV at $90 \mathrm{~J} / \mathrm{m}^{2}$. After $6 \mathrm{~h}, \mathrm{cells}$ were labeled with MitoTracker and immunostained using an anti-Bax antibody. Representative images are shown. Cells with Bax accumulation in mitochondria, indicated by arrows, were scored and are presented as the percentage of total cells in a bar graph. Scale bar: $10 \mu \mathrm{m}$. Data derived from three separate experiments are presented as the means \pm S.E.M. ${ }^{*} P<0.05$ by Student's $t$-test. (i) HeLa cells were transfected with the control vector or pcDNA3-SOCS6, and treated with increasing concentrations of STS for $6 \mathrm{~h}$. Cells were lysed and examined for Bax conformational change as described above. (j) Mock- and HA-SOCS6-transfected HeLa cells were incubated with the crosslinker (DSS) and subjected to western blot analysis using a Bax polyclonal antibody 
recovered in the pellet fractions that were resistant to the extraction as was $\mathrm{Bcl}-2$ (an OMM integral protein) and nicotinamide adenine dinucleotide dehydrogenase (ubiquinone) iron-sulfur protein 3 (NDUFS3, an inner mitochondrial membrane integral protein) (Figure 2f). These data suggest that SOCS6 is tightly associated with the mitochondrial outer membrane.

Ectopic expression of SOCS6 induces mitochondrial fragmentation whereas SOCS6 KD results in elongated mitochondria. Next, we examined the functional consequence of SOCS6 mitochondrial localization. Targeting of ectopic SOCS6 to mitochondria was associated with a concomitant change in mitochondrial morphology from the tubular networks to fragmented puncta in AD293, HeLa, and AZ521 cells (data from AD293 cells shown in Figure 3a). In comparison, ectopically expressed SOCS3 was localized intracellularly, but did not associate with mitochondria (Figure 3a). Thus far, SOCS6 is the only SOCS family protein reported to be located in the mitochondria. Ectopic SOCS6 induced changes in mitochondrial morphology in time- and dose-dependent manners (Figure 3b). In contrast,

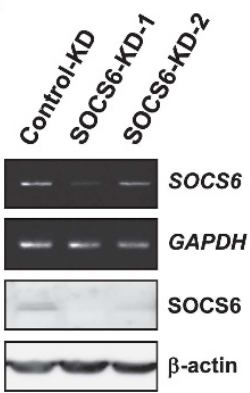

b

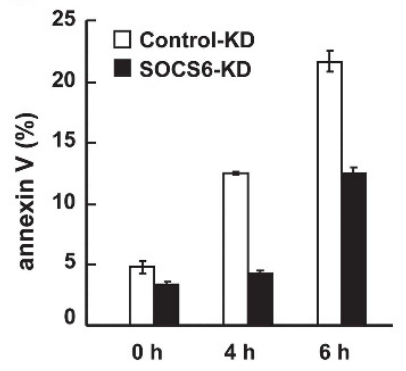

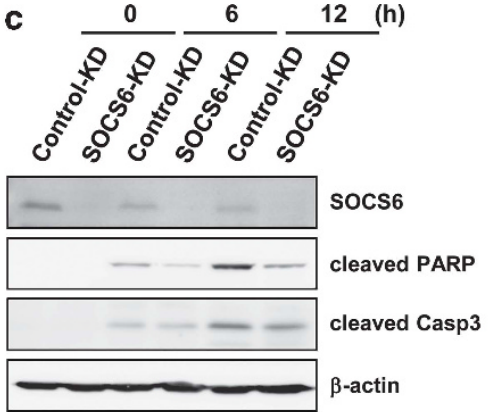

d

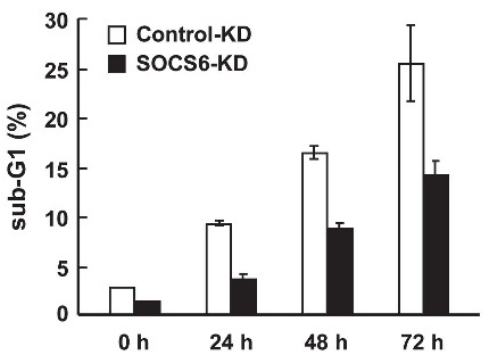

e
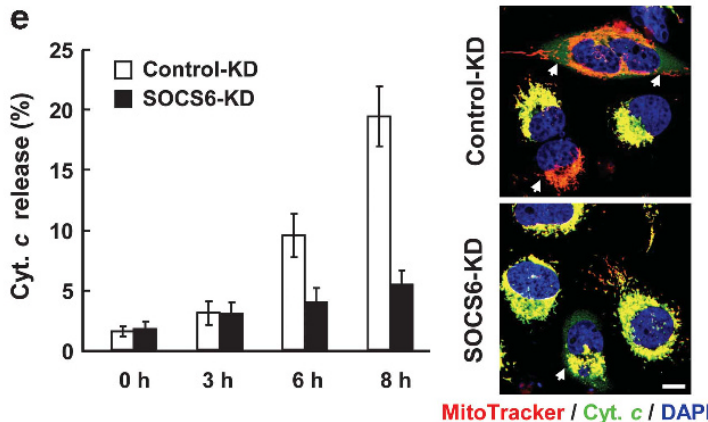

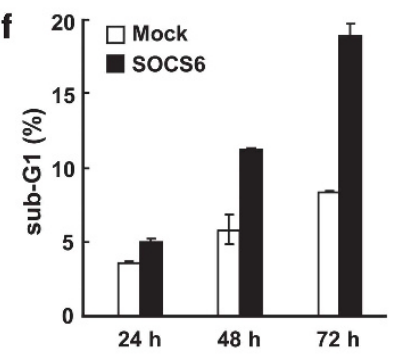

h

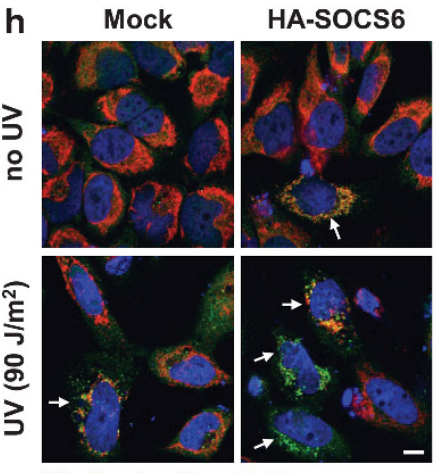

MitoTracker / Bax / DAPI g

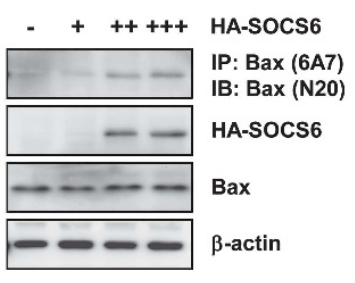

i

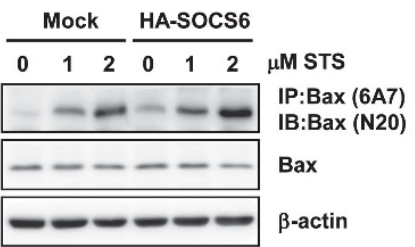

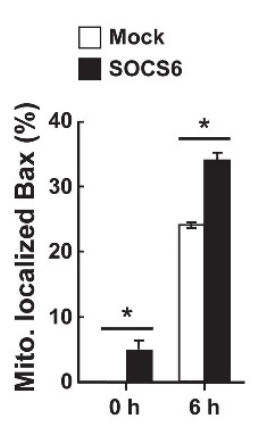

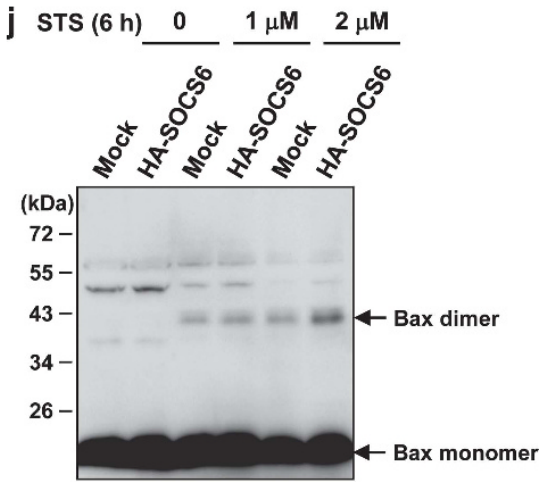



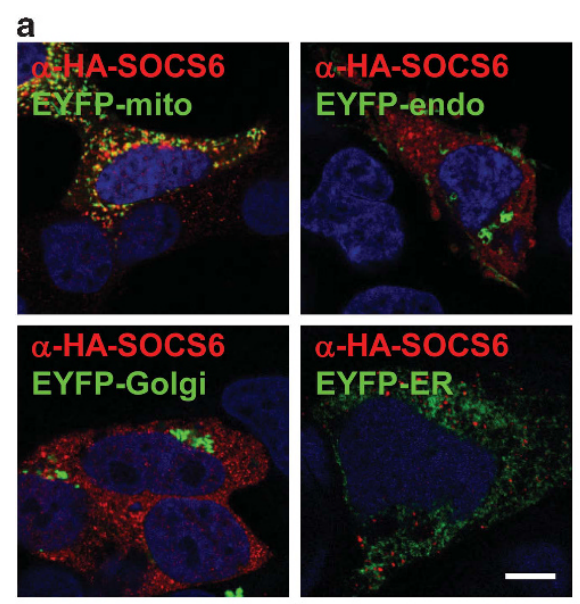

$\begin{array}{lllll}\text { C TCL P1 } & \text { P3 } & \text { P15 P100S100 }\end{array}$

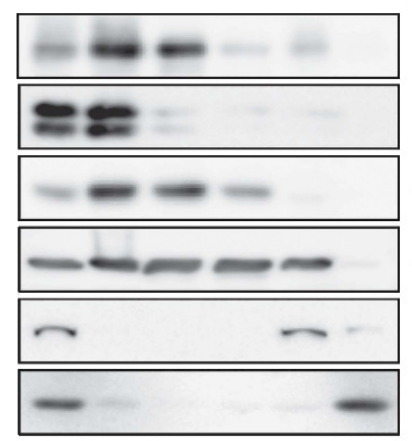

HA-SOCS6

Histone $\mathrm{H} 1$

Complex III

Calnexin

EEA1

14-3-3 $\zeta$
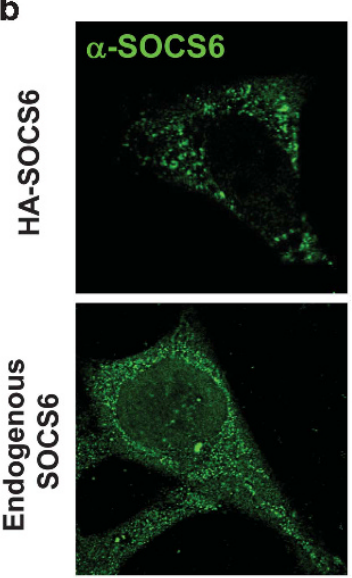
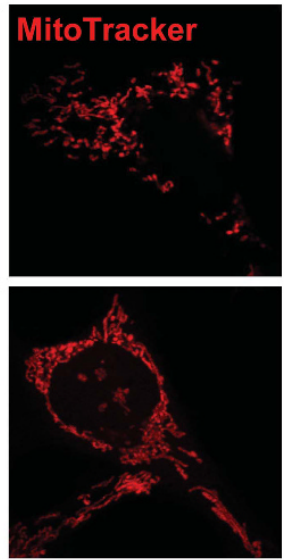
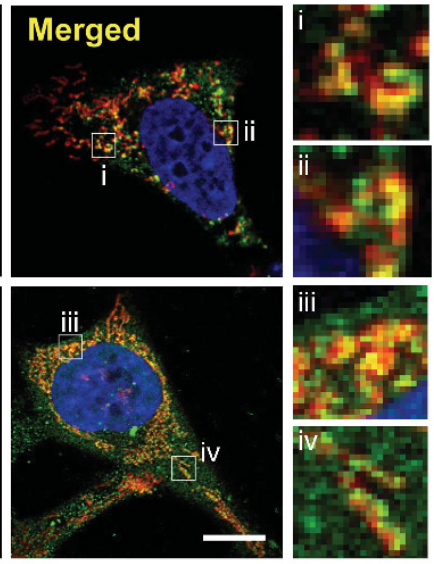

\section{d}

Cyto. Mito. Cyto. Mito.

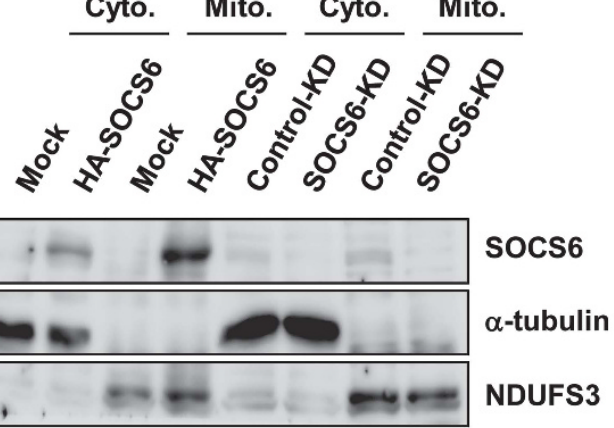

f

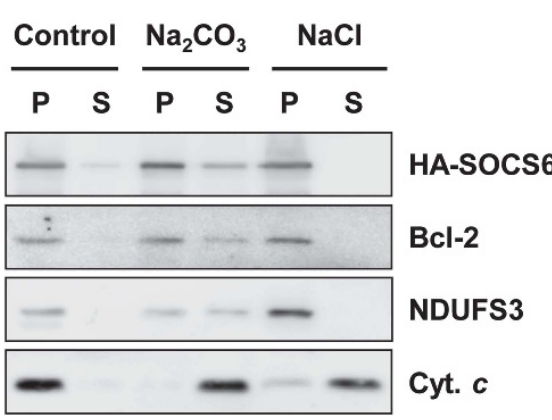

Figure 2 SOCS6 is targeted to the mitochondrial outer membrane. (a) HA-SOCS6 was coexpressed with EYFP-mito, EYFP-endo, EYFP-Golgi, or EYFP-ER in AD293 cells, and examined by immunofluorescence using an anti-HA antibody. Representative images taken by confocal laser microscopy are shown. Scale bar: $10 \mu \mathrm{m}$. (b) Confocal immunofluorescence of ectopic and endogenous SOCS6 (green, anti-SOCS6) in HeLa cells that were loaded with MitoTracker. Scale bar: $10 \mu \mathrm{m}$. Insets show enlarged views of the boxed regions. (c) Total cell lysates (TCLs) were prepared from AD293 cells and subjected to differential centrifugation at $1000 \times g, 3000 \times g$, $15000 \times g$, and $100000 \times g$. Portions of the redissolved particulate fractions (P1, P3, P15, and P100) and the supernatant (S100) derived from the $100000 \times g$ centrifugation were collected and subjected to western blot analysis. (d) Mitochondria were isolated from the mock- and HA-SOCS6-transfected AD293 cells and the control-KD and SOCS6-KD HeLa cells, and subjected to western blot analysis against the indicated proteins. (e) Mitochondria were isolated from AD293 cells transfected with pcDNA3-SOCS6 or the control vector, resuspended in isotonic or hypotonic buffers, and digested with $50 \mu \mathrm{g} / \mathrm{ml}$ trypsin for $30 \mathrm{~min}$, followed by western blot analysis against the indicated proteins. (f) Mitochondria were resuspended in isotonic buffer with or without $0.1 \mathrm{M} \mathrm{Na}_{2} \mathrm{CO}_{3}$ (pH 11.5) or $0.5 \mathrm{M} \mathrm{NaCl}$. After centrifugation at $15000 \times g$ for 10 min, the membrane pellets $(\mathrm{P})$ and supernatants $(\mathrm{S})$ were recovered and subjected to western blot analysis against the indicated proteins

KD of SOCS6 yielded cells with elongated mitochondria (Figure 3c). We further quantified SOCS6-mediated mitochondrial morphological changes using a recently developed Matlab-based automated classification system, Micro-P. ${ }^{27}$ Micro-P classifies mitochondria in a single cell into four categories according to the features of mitochondrial morphology, skeleton, and binary texture, based on the cell micrographs of mitochondria. Mitochondria were sorted in the order of small fragmented mitochondria (type 1), clumped or swollen fragmented mitochondria (type 2), twisted, branched, or circular mitochondria (type 3), and long or linear mitochondria (type 4). According to Micro-P analysis, cells that ectopically expressed SOCS6 displayed a significantly lower percentage of type 4 mitochondrion, with a concomitant increase in type 2. In addition, the average length of the type 3 and type 4 mitochondria in 
a
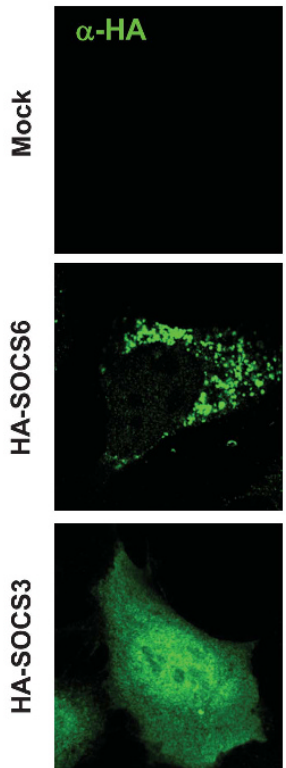

d Mock
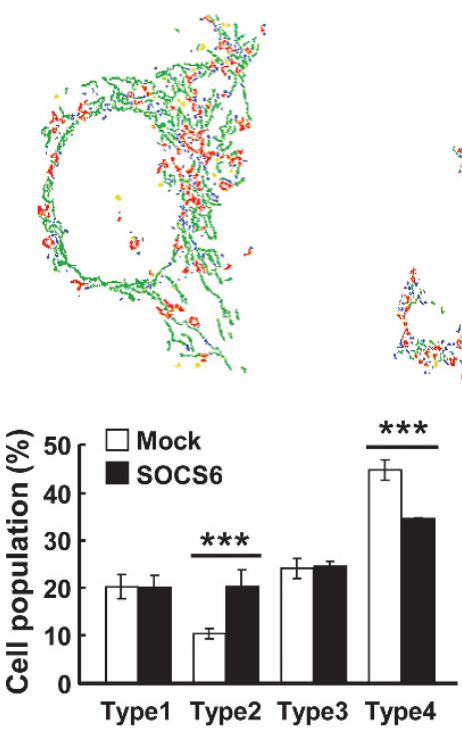

f
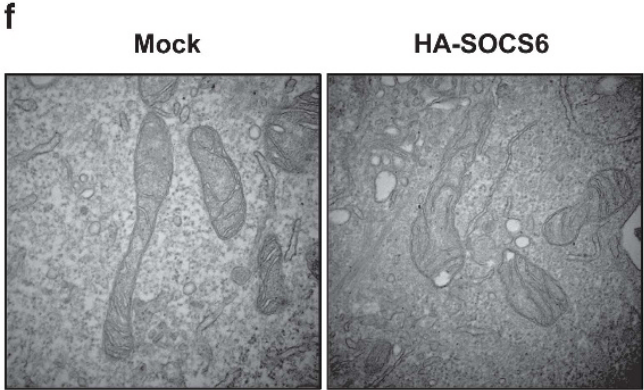

b
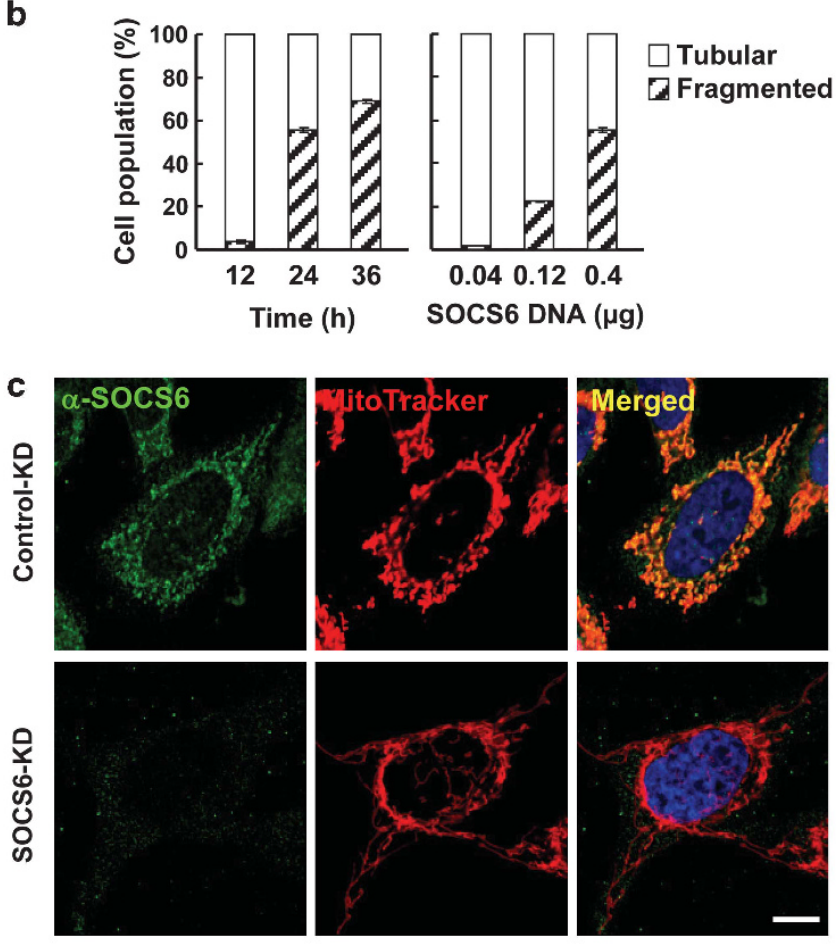

e
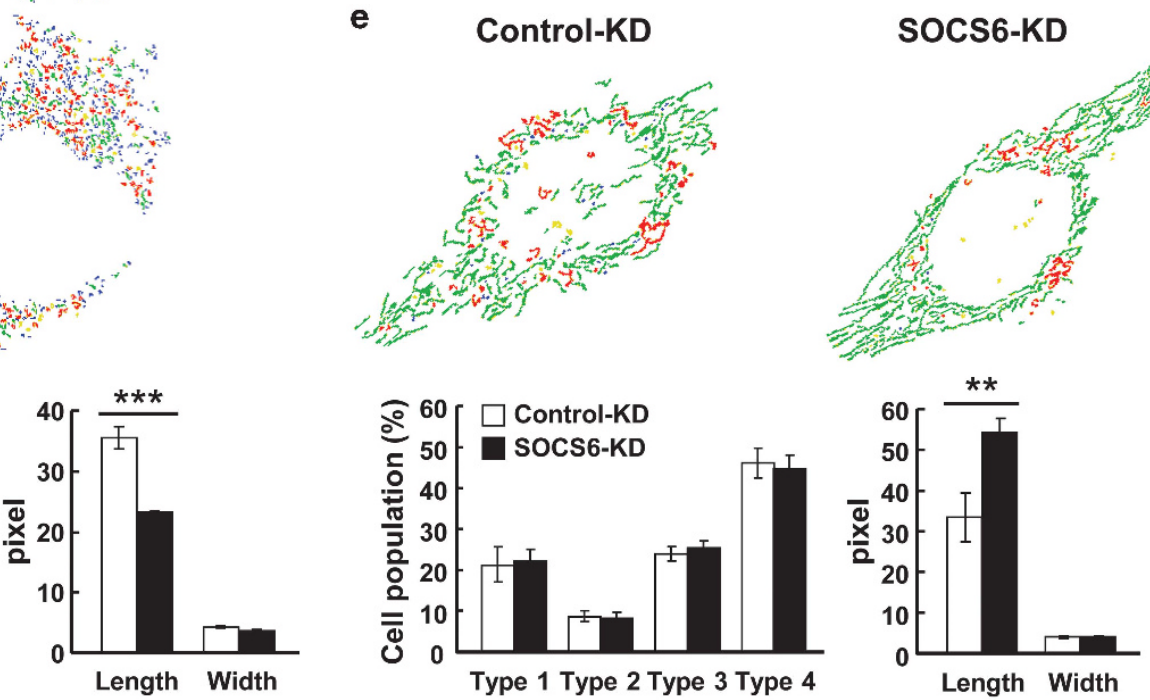

Control-KD

SOCS6-KD
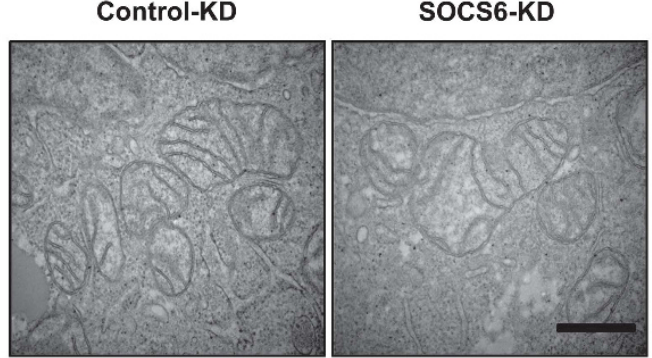

Figure 3 See next page 
SOCS6-expressing cells was significantly shorter than those of the control cells (Figure 3d, lower right panel). We further performed Micro-P analysis of mitochondrial morphology in SOCS6-KD HeLa cells. As shown in Figure 3e, reduction of endogenous SOCS6 by shRNA had little effect on the distribution of the four types of mitochondria; however, the average length of type 3 and type 4 mitochondria was significantly elongated. Electron microscopy revealed that the shortened or elongated mitochondria in SOCS6expressing or $-\mathrm{KD}$ cells retained the intramitochondrial ultrastructure with an intact double-layered membrane and cristae organization (Figure $3 \mathrm{f}$ ). A quantitative analysis was performed to measure the lengths of the cristae membrane (CM) and the inner boundary membrane (IBM), and showed that there was no significant difference in the ratios of $\mathrm{CM} / \mathrm{IBM}$ in SOCS6-overexpressing or -KD cells (Figure 3f).

SOCS6-mediated mitochondrial fragmentation is tightly linked to its targeting to mitochondria. To further define the structural prerequisite for SOCS6-mediated fragmentation, we ectopically expressed a series of SOCS6 mutants in AD293 cells (Figure 4a). Immunofluorescence showed that SOCS6- $\mathrm{N}$ was expressed in the cytosol and the nucleus, whereas SOCS6-C, which retained the $\mathrm{SH} 2$ domain and SOCS box, displayed a punctuate pattern in mitochondria, sharing properties similar to the full-length SOCS6 protein (Figures 4b and c). Notably, SOCS6-C, but not the SOCS6-N mutant, still retained the ability to induce apoptosis (Figure 4d). We further generated a SOCS6 mutant, from which the SOCS box was deleted (SOCS6- $\Delta S B$ ), and showed that SOCS6- $\Delta$ SB failed to target to mitochondria or induce mitochondrial fragmentation (Figures $4 \mathrm{~b}$ and $\mathrm{c}$ ). To determine whether an intact SOCS box is required for targeting SOCS6 to mitochondria, we next generated a SOCS6 mutant in which the terminal helix 3 motif (amino acids 523-535) of the SOCS box was deleted, and showed that this mutant lost mitochondrial localization as well as the ability to induce mitochondrial fragmentation (Figure 4c). These data suggest that SOCS6-mediated mitochondrial fragmentation is tightly linked to its localization to mitochondria, and that an intact SOCS box is required for its localization to mitochondria. In addition, disruption of SOCS box resulted in the loss of its proapoptotic activity (Figure 4d). These data suggested that the SOCS box is requisite for SOCS6 targeting to mitochondria and SOCS6-mediated mitochondrial function.
Manipulation of SOCS6 expression impairs mitochondrial network and function. Mitochondrial networks remain highly dynamic and undergo remodeling through continuous cycles of fission and fusion processes that regulate the tethering of adjacent mitochondria. We further examined the consequence of SOCS6 expression in mitochondrial network dynamics using a polyethylene glycol (PEG) cell fusion assay (Figure 5a). Two HeLa cells, one expressing mitochondriatargeted DsRed (DsRed-mito) and the other expressing mitochondria-targeted EYFP (EYFP-mito), were cocultured and transiently treated with PEG to induce cell fusion. Cyclohexamide $(\mathrm{CHX})$ was included to prevent the synthesis of nascent fluorescent molecules. Apparent fusion of mitochondria was visualized $4 \mathrm{~h}$ after PEG treatment in cell hybrids of the control cells, resulting in mitochondria that carried both EYFP and DsRed. After $7 \mathrm{~h}$, all of the fused cells contained extensively intermixed mitochondria. In contrast, extracellular application of the mitochondrial uncoupling agent carbonyl cyanide $m$-chlorophenylhydrazone (CCCP), which collapses mitochondrial membrane potential $(\Delta \psi \mathrm{m})$, inhibited mitochondrial fusion. Forced expression of SOCS6 also repressed mitochondrial fusion in the cell hybrids.

Next, we performed a quantitative fluorescence recovery after photobleaching (FRAP) assay to measure mitochondrial movement and connectivity in mock- and HA-SOCS6transfected HeLa cells (Figure $5 \mathrm{~b}$ ). Cells harboring fluorescent dye-labeled mitochondria were irradiated by a laser beam to bleach the fluorescence, and the recovery of fluorescence intensity was monitored. The fluorescence of MitoTracker was recovered at a slower rate in SOCS6expressing cells as compared with that of the control cells. These data clearly indicate that SOCS6 expression influences mitochondrial movement, connectivity, and fusion.

The delicate equilibrium between mitochondrial fission and fusion is vital for mitochondrial function. Unbalanced mitochondrial dynamics causing either mitochondrial elongation or excessive fragmentation results in reduced metabolism. ${ }^{28}$ Therefore, we examined whether SOCS6 expression affects mitochondrial function. We first determined whether the $\Delta \psi \mathrm{m}$, a critical aspect of mitochondrial homeostasis, was affected. By measuring mitochondrial permeability transition using the cationic dye JC-1, we show that $\Delta \psi \mathrm{m}$ was significantly lowered in SOCS6-expressing cells and elevated in SOCS6depleted cells (Figure 5c). In parallel, cellular ATP level, lactate production, and glucose uptake were increased in socS6-expressing cells and decreased in SOCS6-depleted

\footnotetext{
Figure 3 SOCS6 regulates mitochondrial morphology. (a) Morphology of mitochondria stained with MitoTracker in AD293 cells transiently transfected with pcDNA3, pcDNA3-SOCS6, or pcDNA3-SOCS3 (green, anti-HA). Scale bar: $10 \mu \mathrm{m}$. (b) AD293 cells were transfected with pcDNA3-SOCS6, and immunofluorescence of HA-SOCS6 was performed after labeling cells with MitoTracker. SOCS6-expressing cells were scored for tubular or fragmented mitochondrial morphology at the designated time points (left panel). Cells in 24 -well plates were transfected with increasing amounts $(0.04,0.12$, and $0.4 \mu \mathrm{g})$ of pcDNA3-SOCS6, and mitochondrial morphology in SOCS6-expressing cells was determined at $24 \mathrm{~h}$ (right panel). A total of 300 SOCS6-expressing cells were examined for each condition in the experiments and data were derived from three independent experiments. (c) Confocal immunofluorescence of SOCS6 (anti-SOCS6) in the control-KD and SOCS6-KD HeLa cells. Scale bar: $10 \mu \mathrm{m}$. (d and e) Micro-P analysis of mitochondrial morphology in HA-SOCS6-expressing AD293 (d) and SOCS6-KD HeLa (e) cells. The Micro-P algorithm classified mitochondria into small fragmented mitochondrion (type 1, blue), large fragmented mitochondrion (type 2, yellow), curved, branched, or circular tubular mitochondrion (type 3, red) and tubular mitochondrion (type 4, green). A representative presentation of Micro-P analysis is shown at the top. Bar graphs at the bottom show the percentages of type 1 to type 4 mitochondria (left panel) and the length and width of type 3 and type 4 mitochondria (right panel) in 300 cells, and are expressed as the means \pm S.E.M. ${ }^{* \star} P<0.01$ and ${ }^{* * *} P<0.001$ by Student's $t$ test. (f) Electron micrographs of mitochondria in AD293 cells transiently transfected with pcDNA3 and pcDNA3-SOCS6 (left panel) and HeLa cells stably harboring the control shRNA-Luc or shRNA-SOCS6 (right panel). Scale bars: $0.5 \mu \mathrm{m}$. Quantitative analysis of intramitochondrial ultrastructure was performed by measuring $\mathrm{CM}$ and IBM length using ImageJ software and the ratio of CM/IBM is shown. A total of 15 mitochondria from each category were scored
} 


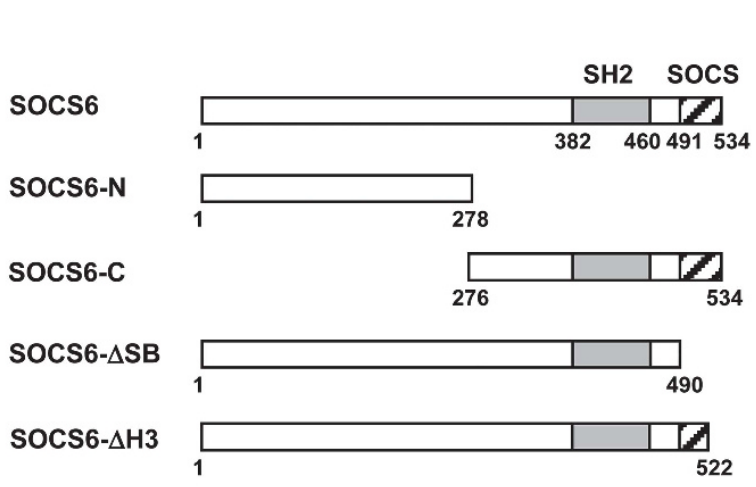

b

Mock
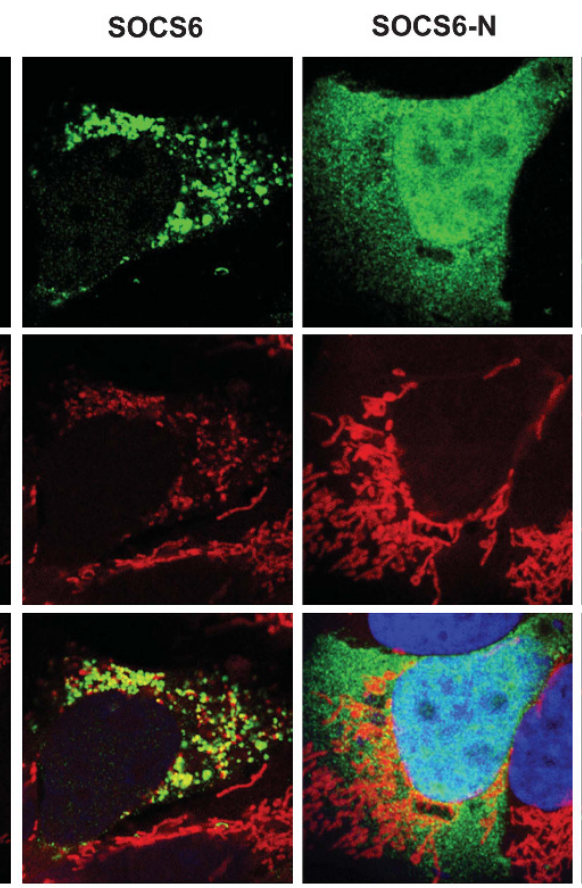

HA-SOCS6 / MitoTracker / DAPI
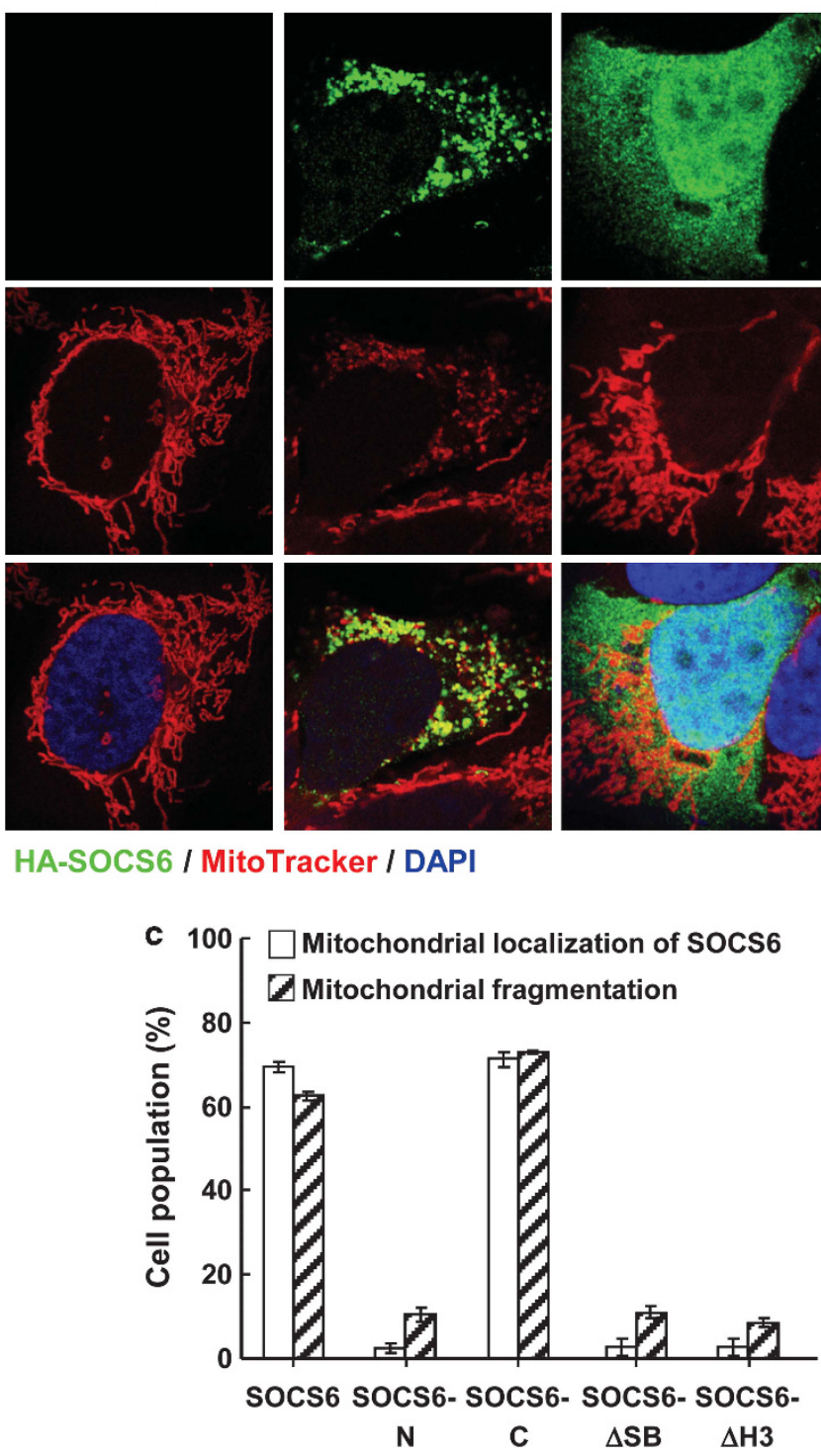

socs6

localization

mito.

cyto./nuc.

mito.

nuc.

cyto.

socs6-C
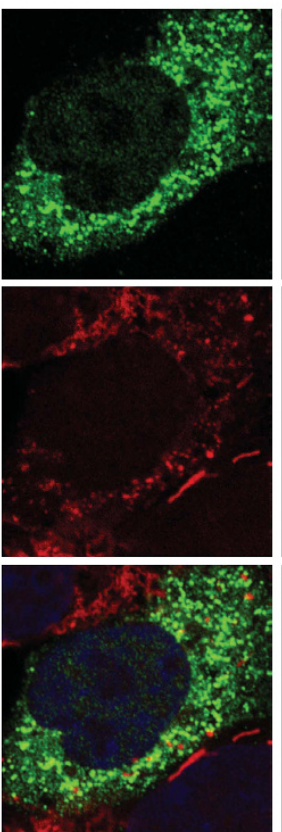

Socs6

pattern

punctate

diffuse

punctate

diffuse

diffuse

\section{Mitochondrial Apoptotic Morphology Sensitization \\ fragmented +

$+$

tubular

fragmented

tubular

tubular
$+$

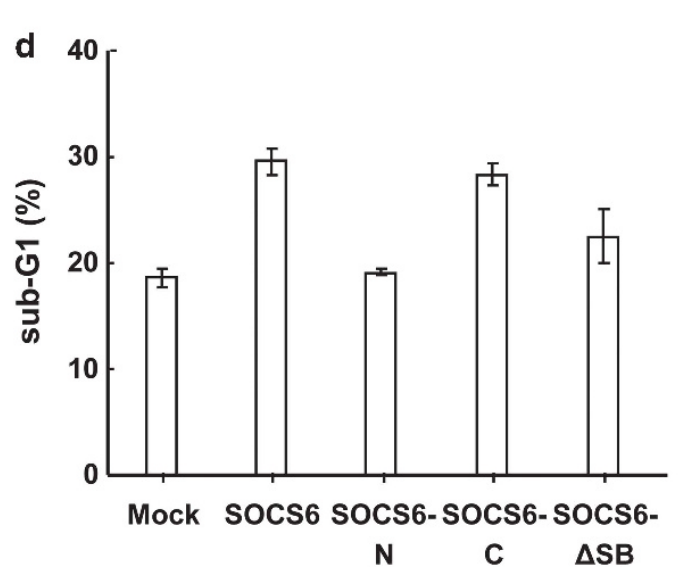

Figure 4 SOCS6-mediated mitochondrial fragmentation is tightly linked to its ability to target to mitochondria. AD293 cells were transfected with pcDNA3 and vectors encoding the full-length and mutant SOCS6 protein. (a) Schematic diagrams of the full-length and mutant SOCS6. The SH2 domain and SOCS6 box are indicated by shaded and hatched boxes, respectively. Mitochondrial localization and distribution pattern of the individual SOCS6 proteins, mitochondrial morphology, and apoptosis are summarized. (b) Immunofluorescence of the full-length and mutant SOCS6 was performed after labeling cells with MitoTracker. Representative confocal images are shown. Scale bar: $10 \mu \mathrm{m}$. (c) Cells were scored for SOCS6 localization and mitochondrial morphology, and are summarized in the bar graphs. A total of 300 transfected cells were counted in each condition, and data were derived from three independent experiments. (d) Cells were subjected to the apoptosis assay of sub-G1 apoptotic fractions by FACS analysis. Data were derived from three separate experiments and are presented as the means \pm S.E.M. 
a

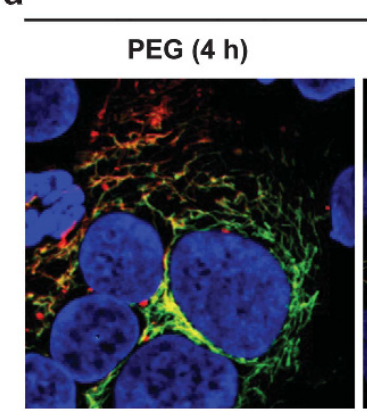

DsRed-mito / EYFP-mito / DAPI
Mock

PEG (7 h)

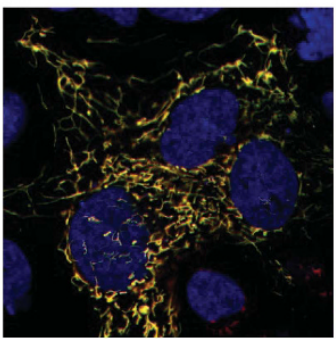

CCCP/PEG (4 h)

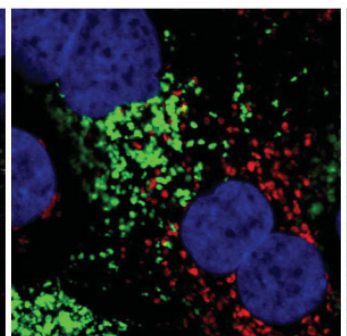

HA-SOCS6

PEG (4 h)

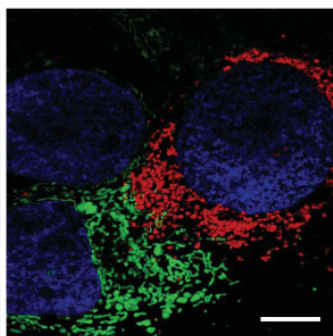

b
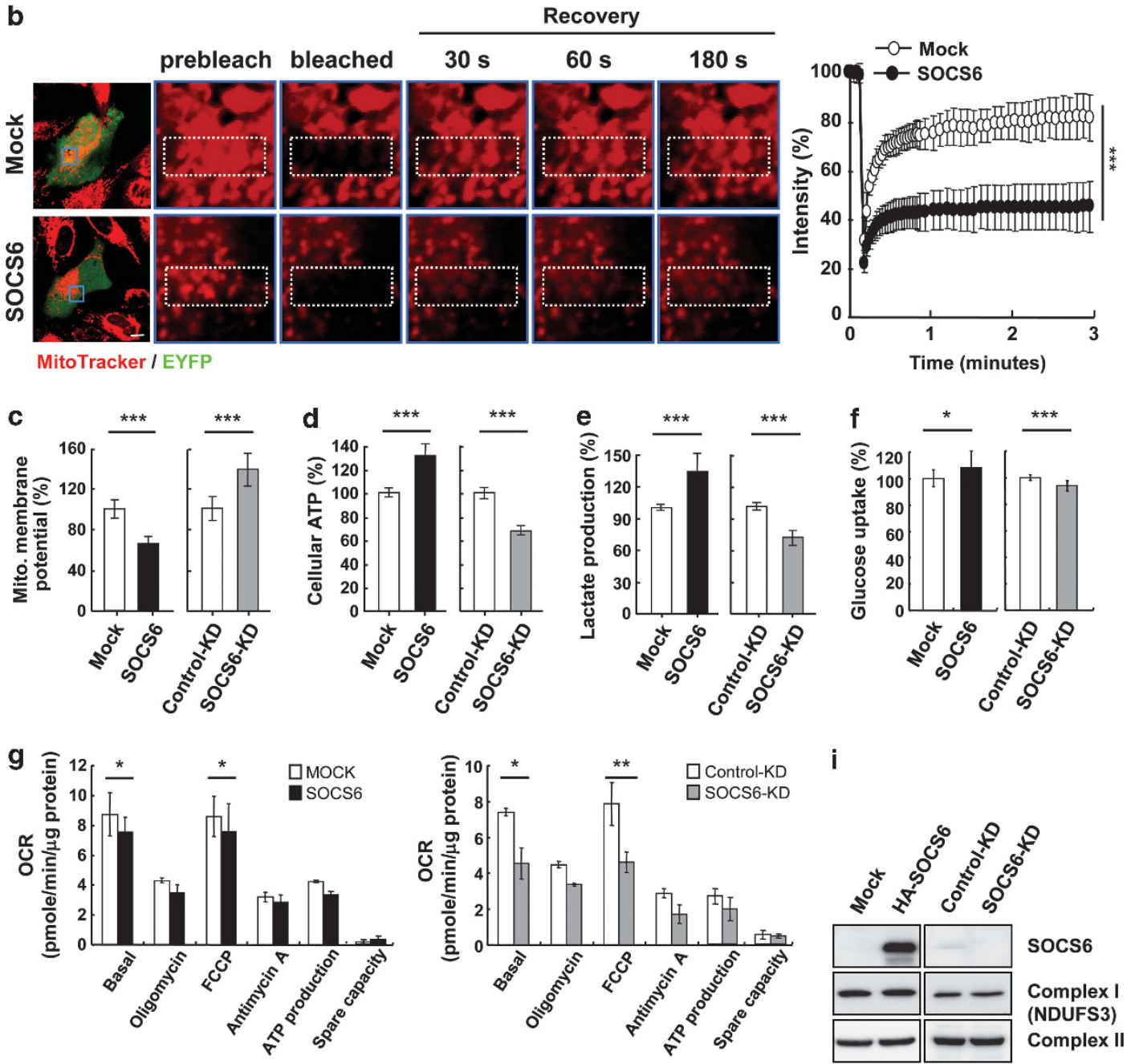

i

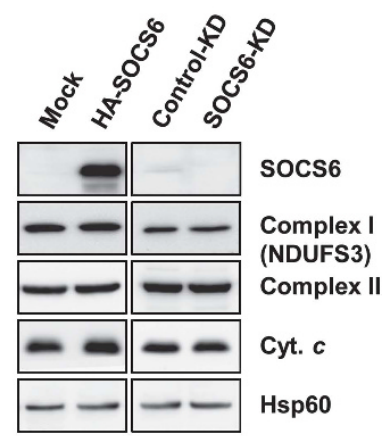

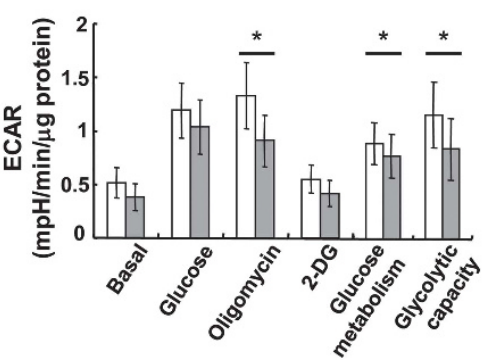


cells (Figures 5d-f). We further examined the effects of SOCS6 expression on mitochondrial respiration and glycolytic function via real-time measurement of the oxygen consumption rate $(\mathrm{OCR})$ and extracellular acidification rate (ECAR). Reduced mitochondrial respiration efficiency was observed in both SOCS6-overexpressing and SOCS6-depleted cells as demonstrated by decreased basal respiration and maximal respiratory capacity (Figure $5 \mathrm{~g}$ ). Moreover, decreased glucose metabolism and glycolytic capacity were noted in the SOCS6-depleted cells (Figure 5h). Nevertheless, expression of mitochondrial proteins related to mitochondrial respiratory complexes remained unchanged in SOCS6-expressing and SOCS6-depleted cells (Figure 5i).

socs6-mediated mitochondrial fragmentation involves DRP1 fission activity. Next, we examined whether SOCS6 induced mitochondrial fragmentation by interfering with fusion and/or fission processes. Overexpression of MFN1 promoted mitochondrial fusion, and hyperaggregated mitochondria were observed in $>90 \%$ of the transfected cells. Coexpression of SOCS6 with MFN1 had little effect on MFN1-elicited mitochondrial fusion (Figures $6 a$ and b). Similarly, SOCS6 expression had a marginal effect on MFN2 fusion activity. Ectopic expression of a dominant-negative form of DRP1 (DRP1-K38A) blocked endogenous DRP1 fission activity, ${ }^{29}$ yielding hyperfused mitochondria in $>90 \%$ of the transfected cells. Coexpression of SOCS6 attenuated DRP1-K38A-elicited hyperfusion (Figures $6 \mathrm{c}$ and d), suggesting cross-modulation between SOCS6 and DRP1 fission activity. Furthermore, inhibition of endogenous DRP1 activity by the dominant-negative mutant alleviated cells from SOCS6-mediated mitochondrial fragmentation, suggesting that SOCS6-mediated fragmentation was dependent on DRP1 fission activity.

To further explore whether SOCS6-induced mitochondrial fragmentation is mediated through DRP1, we employed the shRNA KD approach to lower DRP1 expression. Cells stably harboring DRP1-shRNA displayed hyperfused mitochondria, and ectopic HA-SOCS6 failed to induce mitochondrial fragmentation in DRP1-KD cells (Figures 6e-g), suggesting that SOCS6-induced mitochondrial fragmentation is mediated through DRP1 fission activity.

SOCS6 promotes DRP1 mitochondrial translocation by attenuating its phosphorylation. To establish a direct link between SOCS6 and mitochondrial fission activity, we examined whether SOCS6 affects the expression of mitochondrial dynamic proteins. As shown in Figure 7a, DRP1 protein levels remained unchanged upon overexpression or KD of SOCS6. Similarly, no significant change in MFN1, MFN2, or OPA1 was observed. As DRP1 is shuttled between the mitochondria and cytosol, we further examined whether SOCS6 expression affects its distribution. Ectopic expression of SOCS6 promoted the localization of DRP1 in mitochondria, whereas SOCS6 KD led to a decreased level of mitochondria-localized DRP1 (Figures 7b and c). Co-immunoprecipitation further revealed that ectopic SOCS6 was associated with DRP1 (Figure 7d), and that the association of SOCS6 and DRP1 was readily detected in the mitochondria (Figure 7e). In agreement, endogenous DRP1 partially colocalized with HA-SOCS6, as shown by confocal microscopy (Figure 7f). Phosphorylation of DRP1 has been shown to regulate its mitochondrial translocation and hence its fission activity. ${ }^{16,17}$ Therefore, we examined whether SOCS6 affects DRP1 phosphorylation. Using an antibody specifically recognizing phosphorylated DRP1 at Ser $^{637}$, we showed that phosphorylation of DRP1-Ser ${ }^{637}$ was induced by forskolin and 3-isobutyl-1-methylxanthine (IBMX) treatment (Figures $7 \mathrm{~g}$ and $\mathrm{i}$ ), and that ectopic expression of SOCS6 attenuated DRP1 phosphorylation (Figures $7 \mathrm{~g}$ and h). On the other hand, KD of SOCS6 promoted its phosphorylation (Figures 7i and j).

Wang et al. ${ }^{18}$ recently demonstrated that mitochondrial phosphatase PGAM5 plays an important role in the regulation of programmed necrosis by dephosphorylating DRP1-Ser ${ }^{637}$ and promoting DRP1 mitochondrial translocation. We further examined whether PGAM5 is involved in SOCS6-mediated DRP1 mitochondrial translocation by knocking down PGAM5 expression (Figure 8a). KD of PGAM5 alone led to elongated mitochondria, and ectopic expression of SOCS6 significantly suppressed PGAM5-KD-elicited mitochondrial elongation (Figures $8 \mathrm{~b}$ and $\mathrm{c}$ ). Most importantly, KD of PGAM5 significantly alleviated SOCS6-mediated mitochondrial fragmentation (Figures 8b and c), suggesting that SOCS6induced mitochondrial fission was in part dependent on PGAM5. Co-immunoprecipitation showed that ectopic SOCS6 was associated with both PGAM5-L and PGAM5-S isoforms (Figure 8d). Confocal microscopy confirmed colocalization of HA-SOCS6 with endogenous PGAM5 (Figure 8e). Co-immunoprecipitation revealed that HA-SOCS6 associated in complex with endogenous DRP1 and PGAM5 (Figure 8f). These data suggest that SOCS6 promotes DRP1

Figure 5 SOCS6 impairs mitochondrial function. (a) PEG cell fusion assay. Two HeLa cell clones each containing mitochondrial-targeted red fluorescent protein from Discosoma (DsRed-mito) or EYFP (EYFP-mito) were transiently transfected with the pcDNA3 or pcDNA3-SOCS6. After PEG treatment, confocal images were taken at the indicated time points. Scale bar: $10 \mu \mathrm{m}$. (b) FRAP analysis. HeLa cells were transiently transfected with pIRES-EYFP (mock) or pIRES-EYFP-SOCS6 (SOCS6), and loaded with MitoTracker. Photobleaching of MitoTracker fluorescence was performed in ROls (indicated in white rectangles). Fluorescence images were taken and enlarged images within the blue squares of the prebleached, bleached, and recovered are shown after 30,60, and $180 \mathrm{~s}$. Scale bar: $10 \mu \mathrm{m}$. Relative fluorescence intensity of MitoTracker in ROIs was recorded and plotted during a photobleaching protocol as a function of time $\left(n=20\right.$ cells). Error bars represent the S.E.M. ${ }^{\star \star \star} P<0.001$ by Student's $t$ test. (c- $\left.\mathbf{f}\right)$ AD293 cells transfected with pcDNA3 (mock) or pcDNA3-SOCS6 (SOCS6) (left panels), and HeLa cells stably harboring lentivirus-based shRNA targeting luciferase or SOCS6 (right panels) were measured for mitochondrial membrane potential $(\Delta \psi \mathrm{m})$ using the cationic dye JC-1 by flow cytometry (c), total cellular ATP level (d), lactate production (e), and glucose uptake (f). Percentage shown relative to the control cells were derived from three separate experiments and are presented as the means \pm S.E.M. ${ }^{* \star *} P<0.001$ by Student's $t$-test. ( $\mathbf{g}$ and $\mathbf{h}$ ) The mock- and HA-SOCS6-transfected HeLa cells and the control-KD and SOCS6-KD HeLa cells were subjected to real-time measurement of OCR $(\mathbf{g})$ and ECAR $(\mathbf{h})$ using an Extracellular Flux Analyzer. OCR was measured at steady state followed by sequential incubation with $3 \mu \mathrm{M}$ oligomycin, $1 \mu \mathrm{M} \mathrm{FCCP}$, and $5 \mu \mathrm{M}$ antimycin A. ECAR was measured at steady state followed by sequential incubation with $25 \mu \mathrm{M}$ glucose, $3 \mu \mathrm{M}$ oligomycin, and $50 \mu \mathrm{M} 2-\mathrm{DG}$. Data were derived from three separate experiments and are presented as the means \pm S.E.M. ${ }^{\star} P<0.05$, and ${ }^{* \star} P<0.01$ by Student's t-test. (i) Total cell lysates were prepared and subjected to immunoblotting against the indicated proteins 
mitochondrial localization in part by forming complex with DRP1 and PGAM5, and attenuates DRP1 phosphorylation.

\section{Discussion}

In this study, we demonstrated that SOCS6 encodes a mitochondria targeting protein regulating mitochondrial morphology and apoptosis. Ectopic expression of SOCS6 induced extensive mitochondrial fragmentation, whereas silencing SOCS6 led to elongated mitochondria. SOCS6 promoted mitochondrial fragmentation in part through forming complex with DRP1 and PGAM5, attenuating DRP1 phosphorylation, and promoting DRP1 mitochondrial translocation. Concomitant with mitochondrial fragmentation, ectopic SOCS6 elicited Bax activation and apoptosis. In accordance with our previous findings that SOCS6 was frequently downregulated in gastric adenocarcinoma and ectopic expression of SOCS6 inhibited colony formation, we showed in this study that loss of SOCS6 delayed cytochrome $c$ release and suppressed apoptosis.

SOCS6-mediated mitochondrial fragmentation could be due to reduced fusion, enhanced fission, or both. Mitochondrial fusion is initiated by mitofusin-mediated mitochondrial tethering. MFN1 and MFN2 form both homotypic and heterotypic complexes. ${ }^{9}$ SOCS6, MFN1, and MFN2 are all OMM proteins. However, SOCS6 does not interfere with mitofusin complex formation (data not shown) or affect mitofusin-mediated fusion (Figures $6 a$ and b). In contrast, inhibition of DRP1 activity by a dominant-negative DRP1 or by shRNA-KD approach conferred cells increased resistance to SOCS6-mediated mitochondrial fragmentation (Figures 6d and g). These data suggest that SOCS6-induced mitochondrial fission is dependent on DRP1 activity. In support, we showed that ectopic SOCS6 promoted DRP1 mitochondrial localization by forming complexes with DRP1 and mitochondrial phosphatase PGAM5, leading to decreased DRP1-Ser ${ }^{637}$ phosphorylation (Figures 7 and 8). However, KD of PGAM5 significantly suppressed but not completely abrogated SOCS6-mediated mitochondrial fragmentation (Figure 8c), suggesting that activities other than that of PGAM5 are involved in SOCS6-mediated fragmentation.

Shifting the mitochondrial dynamic equilibrium toward fission or fusion by up- or down-regulation of proteins controlling fission and fusion processes confers to cells an increased sensitivity or resistance toward apoptosis. ${ }^{30}$ The mitochondrial targeting proteins endophilin $\mathrm{B} 1^{31}$ and multiple indicator cluster survey 1 (MICS1) ${ }^{32}$ regulate mitochondrial morphology and affect apoptosis. Ectopic expression of MICS1 induced mitochondrial elongation, and delayed cytochrome $c$ release and the progression of apoptosis. ${ }^{32}$ Endophilin B1, which is required for the modulation of normal mitochondrial outer membrane morphological dynamics, promoted Bax activation. ${ }^{31}$ In this study, we demonstrate that SOCS6 is a novel mitochondrial targeting protein that promotes mitochondrial fission. In addition, we showed that ectopic SOCS6 facilitated UV-induced apoptosis (Figure 1f), suggesting that cells expressing ectopic SOCS6 exhibited a lower threshold against apoptotic insults because of the reduced $\Delta \psi \mathrm{m}$. Furthermore, ectopic SOCS6 induced Bax activation (Figure 1g) and cell death (data not shown) in a dose-dependent manner, supporting the notion that mitochondrial fragmentation actively participates in apoptosis.

By immunofluorescence and biochemical analyses, we showed that SOCS6 is tightly associated with mitochondria exposed to the cytosol (Figure 2). However, a putative mitochondrial targeting sequence was not detected in the $\mathrm{N}$-terminus of SOCS6. The fact that the SOCS6-C mutant, which contains only the C-terminal half of the SOCS6 protein, mimicked the full-length protein to be localized to mitochondria (Figure 4) suggested the possibility of an internal mitochondrial targeting signal. Alternatively, posttranslational modification of proteins with glycosylphosphatidylinositol (GPI) moiety allows the anchorage of proteins to lipid bilayers. Using big-PI predictor for GPI modification site prediction, we identified a potential GPI modification site at the C-terminus of SOCS6 at $A s n^{524}$. Mutagenesis was performed to generate a SOCS6-N524L mutant allele. Our data showed that the SOCS6-N524L mutant was no longer localized to mitochondria (data not shown). To our knowledge, SOCS6 is the first SOCS family protein reported to be located in the mitochondria. Further investigation is warranted to study the targeting of SOCS6 to mitochondria.

Like all SOCS family proteins, SOCS6 contains a central $\mathrm{SH} 2$ domain and a C-terminal SOCS box. The SOCS box was reported to act as a common binding domain for a large family of cullin-RING-based E3 ubiquitin protein ligase complexes. ${ }^{33}$ By functioning as an adaptor protein, SOCS protein links substrates to the Elongin BC-Cul2/Cul5-SOCS box protein (ECS) E3 ligase complexes for ubiquitylation and degradation. ${ }^{34}$ Recently, several E3 ligases were reported to regulate mitochondrial dynamics through ubiquitylation and proteasomal degradation. ${ }^{35} \mathrm{MARCH}-\mathrm{V}$, membrane-associated ring finger (C3HC4) 5 (also called MITOL), bound to MFN2 and DRP1 and promoted ubiquitylation of DRP1. ${ }^{36}$ Parkin, an IBR-domain E3 ligase, induced the ubiquitylation and degradation of MFN1 and MFN2, and interacted with and ubiquitylated DRP1, thus promoting its degradation. ${ }^{37}$ In our study, we showed that an intact SOCS box is a prerequisite for SOCS6 mitochondrial localization and SOCS6-mediated mitochondrial function. According to X-ray crystallographic data, ${ }^{34-36}$ the SOCS box is a conserved structure fold with three core helices that mediate the interaction with other adaptor proteins of the ECS E3 ligase complexes. We showed that deletion of the $\mathrm{H} 3$ helix, which is believed to disturb the stability of SOCS box folding, abolished SOCS6-mediated mitochondrial fission and apoptosis (Figure 4). SOCS6 was shown to act as a component of the ECS E3 ligase complex, interacting with c-KIT and regulating its ubiquitylation and turnover. ${ }^{38}$ Whether ECS-mediated protein ubiquitylation and degradation plays a role in SOCS6-mediated mitochondrial fragmentation and apoptosis remains to be determined.

In summary, we showed that SOCS6 is a novel mitochondrial morphogenic factor and an important component of the mitochondrial pathway for apoptosis. Our data showed that loss of SOCS6 confers cells increased antiapoptotic activity to cells, corroborating the important role of mitochondrial dynamics in malignant transformation and cancer formation. These results will allow us to investigate the role of SOCS6mediated mitochondrial morphogenesis in other pathologies, 


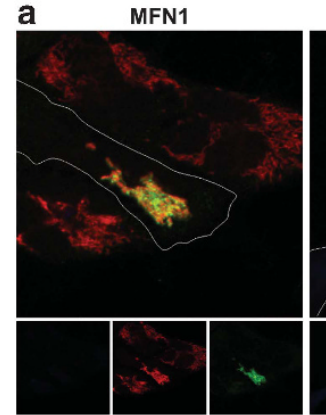

SOCS6 / MitoTracker / MFN1

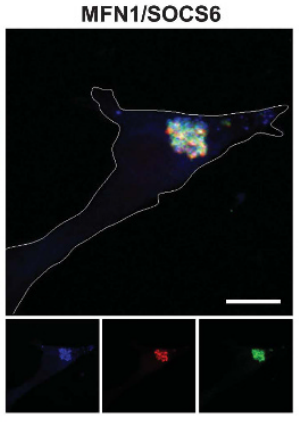

C

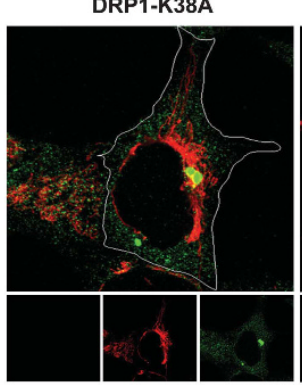

Socs6 / MitoTracker / DRP1

DRP1-K38A/SOCS6

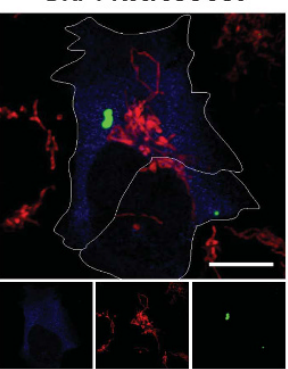

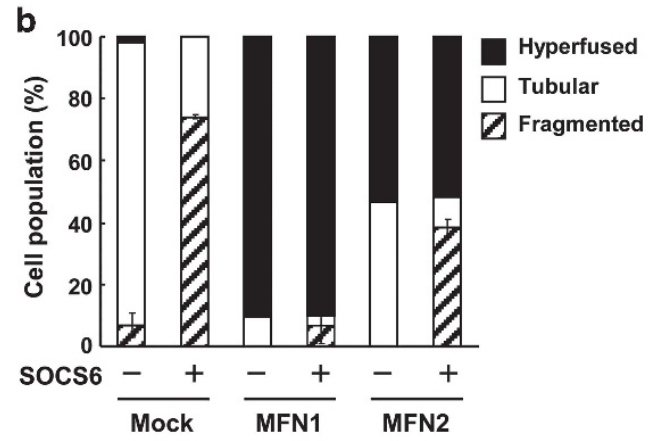

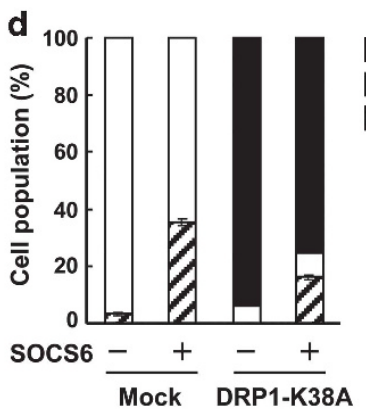

e

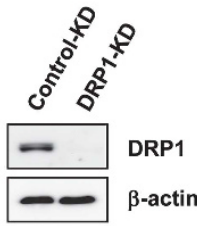

f

f

Control-KD

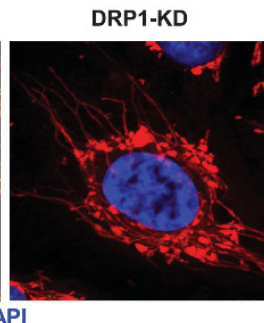

DRP1 / MitoTracker / DAP I

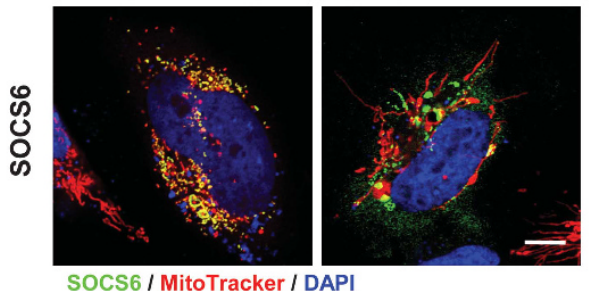

g

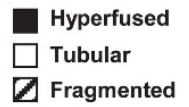

Hyperfused $\square$ Tubular $\triangle$ Fragmented

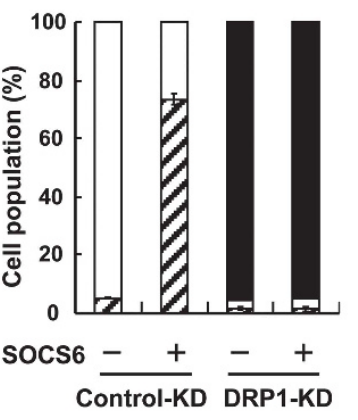

Figure 6 SOCS6-mediated mitochondrial fragmentation is dependent on DRP1 fission activity. (a-d) AD293 cells were transfected with vectors encoding MFN1-myc, MFN2-myc, or DRP1-K38A alone or with pCDNA3-SOCS6. Immunofluorescent staining was performed after labeling cells with MitoTracker. Representative confocal images of HA-SOCS6 (blue, anti-SOCS6), MitoTracker, and MFN1-myc (green, anti-myc) (a) or DRP1-K38A (green, anti-DRP1) (c), are displayed at the bottom, whereas the top displays the merged, enlarged view. Scale bar: $10 \mu \mathrm{m}$. (b and d) Transfected cells were scored according to their mitochondrial morphology as tubular networks, hyperfused, or fragmented patterns. Percentages of cells with designated mitochondrial patterns are indicated in the bar graph. A total of 300 cells were counted for each category and data were derived from three independent experiments. (e-g) HeLa cells stably harboring shRNAs targeting luciferase (control-KD) and DRP1 (DRP1-KD) are shown. (e) DRP1 expression was determined by western blot analysis. (f) Confocal images of MitoTracker and DRP1 (green, anti-DRP1) or SOCS6 (green, anti-SOCS6) are shown in upper or bottom panel. Scale bar: $10 \mu \mathrm{m}$. (g) Transfected cells were scored according to their mitochondrial morphology as tubular networks, hyperfused, or fragmented patterns, and the percentages of distribution are shown in the bar graph. A total of 300 cells were counted for each category and data were derived from three independent experiments

including neurodegenerative and cardiovascular diseases and diabetes.

\section{Materials and Methods}

Cell culture and transfection. AD293, a derivative of HEK293 cell line with improved cell adherence, ${ }^{39}$ and HeLa cells were grown in Dulbecco's modified Eagle's medium (DMEM) supplemented with 10\% fetal bovine serum (FBS) at $37^{\circ} \mathrm{C}$ in a humidified atmosphere of $5 \% \mathrm{CO}_{2}$. Transient transfections were performed using Lipofectamine 2000 (Invitrogen, Carlsbad, CA, USA) or
TurboFect (Fermentas, Glen Burnie, MD, USA) according to the instructions of the manufacturers.

Antibodies. The SOCS6-N recombinant protein (amino acids 1-278) was expressed in Escherichia coli BL21 (DE3) pLysS cells carrying pET21-SOCS6-N, and gel-purified to raise antibodies in rabbits. Anti-SOCS6 serum was affinitypurified by incubation with nitrocellulose membrane strips to which the same recombinant protein was bound, followed by elution with $0.2 \mathrm{M}$ glycine $(\mathrm{pH} 2.7)$ and immediate neutralization using $1 \mathrm{M}$ Tris ( $\mathrm{pH} 8.0)$. Anti-cleaved PARP (1: 1000; 
a

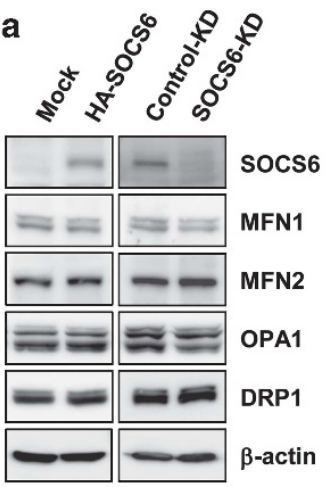

b

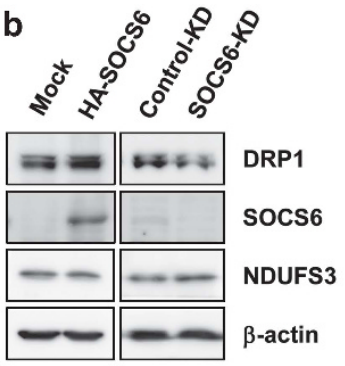

Mitochondrial fractions c

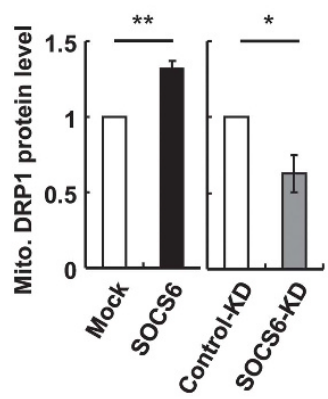

d
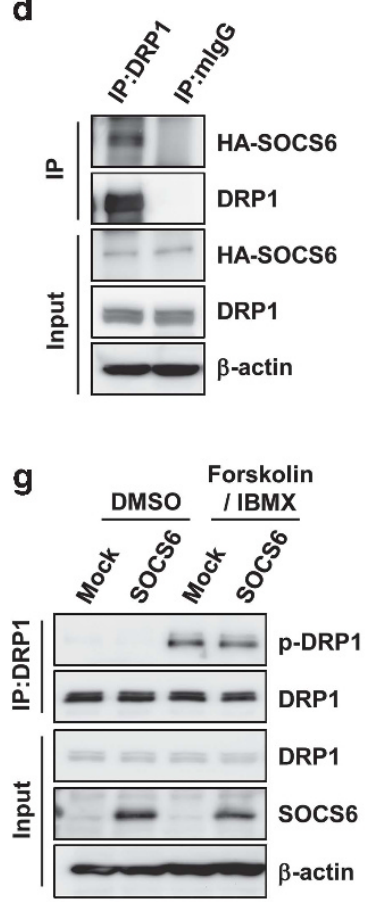

e

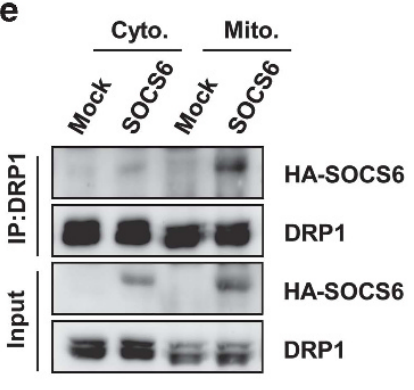

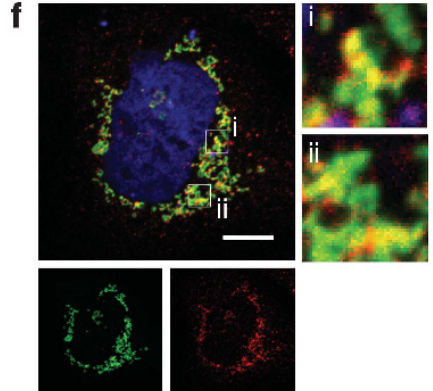

SOCS6 / DRP1 / DAPI
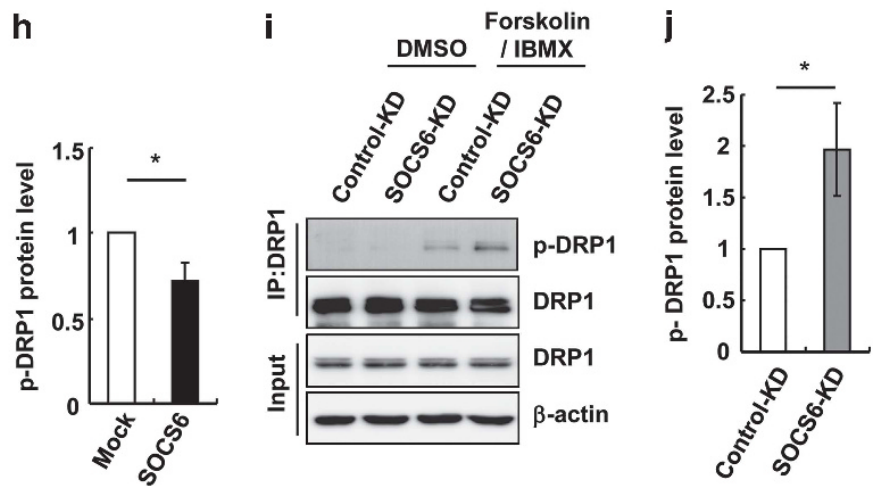

Figure 7 SOCS6 forms a complex with DRP1, attenuates DRP1 phosphorylation, and promotes DRP1 mitochondrial translocation. HeLa cells transiently transfected with pcDNA3 or pcDNA-SOCS6 and the cells stably harboring lentivirus-based shRNA targeting luciferase or SOCS6 were examined. (a) Total cell lysates were prepared and subjected to immunoblotting against the indicated proteins. (b and $\mathbf{c}$ ) Mitochondria were purified and subjected to western blot analysis (b). The bar graph in (c) shows the quantification of the DRP1 protein level in the mitochondrial fraction. Data were derived from three separate experiments and are presented as the means \pm S.E.M. ${ }^{*} P<0.05$ and ${ }^{* \star} P<0.01$ by Student's $t$-test. (d) Cell lysates were prepared and subjected to immunoprecipitation with the control IgG or anti-DRP1 beads followed by western blot analysis of the immunoprecipitates using the indicated antibodies. (e) The cytosolic and mitochondrial fractions were isolated and subjected to immunoprecipitation followed by western blot analysis. (f) Confocal images of HA-SOCS6 (green, anti-SOCS6) and endogenous DRP1 (red, anti-DRP1) in HeLa cells expressing HA-SOCS6. Scale bar: $10 \mu \mathrm{m}$. Insets show enlarged views of the boxed areas. ( $\mathbf{g}-\mathbf{j})$ Mock- or HA-SOCS6-expressing cells in ( $\mathbf{g}$ and $\mathbf{h}$ ) and control-KD or SOCS6-KD cells in (i and $\mathbf{j})$ were incubated in the presence or absence of $20 \mu \mathrm{M}$ forskolin and $10 \mu \mathrm{M} \mathrm{IBMX}$ for $1 \mathrm{~h}$, and subjected to immunoprecipitation using a DRP1 antibody followed by immunoblotting against a phospho-DRP1(Ser637) antibody and the indicated antibodies in ( $\mathbf{g}$ and $\mathbf{~})$. Bar graphs in ( $\mathrm{h}$ and $\mathbf{j}$ ) show the quantification of phospho-DRP1 protein levels in cells treated with or without forskolin and IBMX. Data were derived from three separate experiments and are presented as the means \pm S.E.M. ${ }^{*} P<0.05$ by Student's $t$-test

BD Biosciences, Franklin Lakes, NJ, USA), anti-cleaved caspase-3 (1:100; Millipore, Billerica, MA, USA), anti- $\beta$-actin (1:5000; Sigma, St. Louis, MO, USA), anti-HA (1: 1000; Covance, Princeton, NJ, USA), anti-Bax (1:1000, N-20; Santa Cruz Biotechnology, Santa Cruz, CA, USA), anti-complex III (1:1000; Invitrogen), anti-calnexin (1:1000; Santa Cruz Biotechnology), anti-EEA1 (1:1000; BD Biosciences), anti-14-3-3- $\zeta$ (1:1000; Santa Cruz Biotechnology), anti-Bcl-2 (1: 1000; Dako, Glostrup, Denmark), anti-Hsp60 (1: 1000; Santa Cruz Biotechnology), anti-complex I NDUFS3 (1:1000; Invitrogen), anti-cytochrome c (1:1000; $\mathrm{BD})$, anti-complex II $70 \mathrm{kDa}(1: 2000$; Invitrogen), anti-DRP1 (1:2000; BD Biosciences), anti-MFN1 (1:1000; Abnova, Taipei, Taiwan), anti-MFN2
(1:1000; Calbiochem, San Diego, CA, USA), anti-OPA1 (1:1000; BD Biosciences), anti-PGAM5 (1: 1000; Sigma), and anti-Parkin (1: 1000; Upstate Biotechnology, Lake Placid, NY, USA) were used. Secondary antibodies included the peroxidase-conjugated anti-mouse, anti-rabbit, and anti-goat IgG (Santa Cruz Biotechnology) in a 1:5000 dilution.

RNA preparation and reverse transcriptase-PCR (RT-PCR). Total RNA was prepared from cells, converted to CDNA, and subjected to RT-PCR as described previously. ${ }^{40} \mathrm{PCR}$ was performed using primers for SOCS6 transcripts (NM_004232; sense primer, 5'-AAGAATTCATCCCTTGGATTAGGTAAC-3'; and 
a

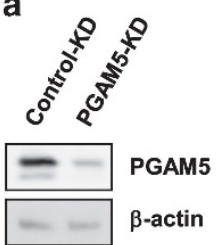

d

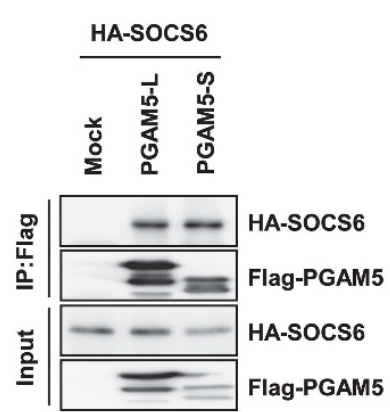

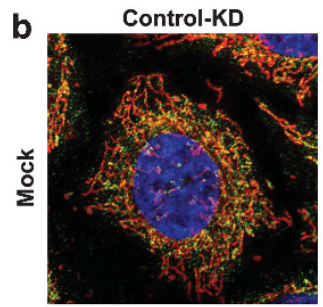

PGAM5-KD

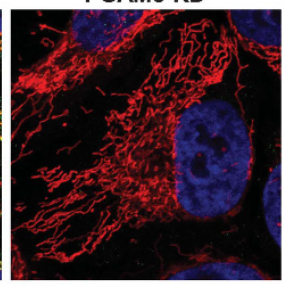

PGAM5 / MitoTracker / DAPI
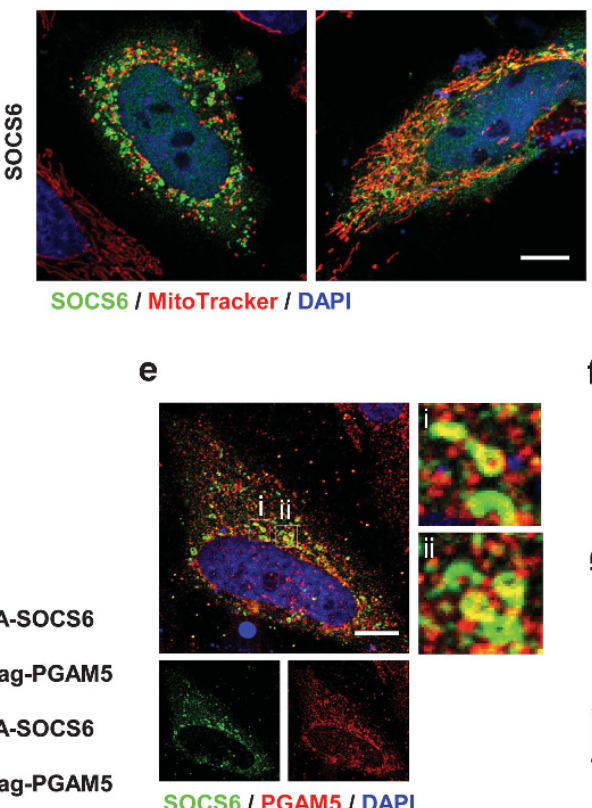

C
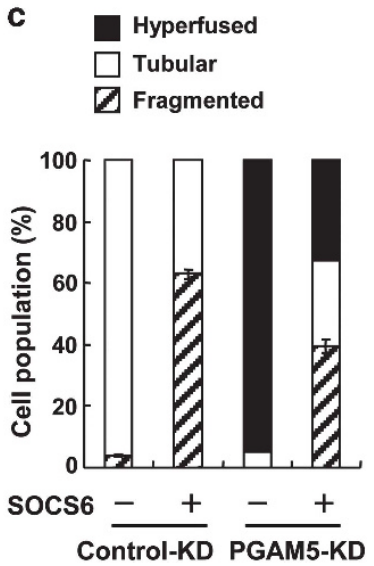

Control-KD PGAM5-KD

Figure 8 socS6-mediated mitochondrial fission is in part mediated by PGAM5 activity. (a-c) HeLa cells stably harboring shRNAs targeting at luciferase (control-KD) and PGAM5 (PGAM5-KD) were established. (a) Total cell lysates were prepared and PGAM5 expression was determined by western blot analysis. (b) Control-KD and PGAM5-KD cells were transiently transfected with pcDNA3 or pcDNA3-HA-SOCS6, and confocal images of MitoTracker and PGAM5 (green, anti-PGAM5) or SOCS6 (green, anti-HA) are shown. Scale bar: $10 \mu \mathrm{m}$. (c) Transfected cells in (b) were scored according to their mitochondrial morphology as tubular networks, hyperfused, or fragmented patterns, and the percentages of distribution are shown in the bar graph. A total of 300 cells were counted for each category and data were derived from three independent experiments. (d) AD293 cells were transiently transfected with pcDNA3-HA-SOCS6 alone or with pcDNA3-Flag-PGAM5-L or pcDNA3-Flag-PGAM5-S, and subjected to immunoprecipitation with the anti-Flag beads followed by western blot analysis of the immunoprecipitates using the indicated antibodies. (e) Confocal images of HASOCS6 (green, anti-SOCS6) and endogenous PGAM5 (red, anti-PGAM5) in HeLa cells expressing HA-SOCS6. Scale bar: $10 \mu \mathrm{m}$. Insets show enlarged views of the boxed areas. (f) Cell lysates were prepared and subjected to immunoprecipitation with the control IgG, anti-DRP1, or anti-PGAM5 beads followed by western blot analysis of the immunoprecipitates using the indicated antibodies

antisense primer, 5'-CAGACTGGAGGTCGTGGAA-3') and glyceraldehyde-3phosphate dehydrogenase (GAPDH) transcripts (NM_002046; sense primer, 5'-TGGTATCGTGGAAGGACTCATGAC-3'; and antisense primer, 5'-ATGCCAGT GAGCTTCCCGTTCAGC-3').

Lentiviral preparation, viral infection, and stable cell generation. The pLKO.1-shRNA plasmids encoding shRNAs with sequences targeting human SOCS6 were purchased from the National RNAi Core Facility (Taipei, Taiwan). The shRNA sequence of shRNA-SOCS6-1 contained 5'-AACCCTAAGTAAAGTT GAATGAA-3' (TRCN0000005499), and shRNA-SOCS6-2 contained 5'-ATAAGTC CAATAACGACAGGC-3' (TRCN0000005500). The shRNA sequence of shRNADRP1 contained 5'-CGGTGGTGCTAGAATTTGTTA-3' (TRCN0000001098). The shRNA sequence of shRNA-PGAM5 contained 5'-GCGCCATAGAGACCACCGAT AT-3' (TRCN0000245651). The shRNA targeting firefly luciferase $5^{\prime}$-CAAATCACAGAATCGTCGTAT-3' (TRCN0000072246) was incorporated as a control. Viruses were collected from the medium $60 \mathrm{~h}$ after transfection. For KD experiments, HeLa cells were infected with the collected viruses over $24 \mathrm{~h}$ in the presence of polybrene, followed by selection in medium containing puromycin $(0.5 \mu \mathrm{g} / \mathrm{ml})$ for $7-9$ days as described previously. ${ }^{41}$
Apoptosis assay. After UV irradiation, cells were harvested at the designated time points, stained with $50 \mathrm{mg} / \mathrm{ml}$ of propidium iodide (PI) for $30 \mathrm{~min}$ at room temperature, and subjected to flow cytometric analysis of sub-G1 apoptotic fractions (FACSCalibur; Becton Dickinson, San Jose, CA, USA). Apoptotic cells were also measured using the annexin-V-FLOUS staining kit (Roche, Basel, Switzerland) for externalized phosphatidylserine.

Bax activation. Cells were lysed in 1\% CHAPS buffer containing protease inhibitors. Immunoprecipitation was performed by incubating protein lysates with an anti-Bax monoclonal antibody (6A7; BD Biosciences) at $4{ }^{\circ} \mathrm{C}$ for overnight. Immunocomplexes in protein lysates were precipitated with Dynabeads (Invitrogen) and subjected to western blot analysis. For detecting Bax oligomerization, cells were washed with phosphate-buffered saline (PBS) and treated with $10 \mathrm{mM}$ of the crosslinking agent disuccinimidyl suberate (DSS) (Sigma) in dimethylsulfoxide (DMSO) at room temperature for $30 \mathrm{~min}$. After quenching the crosslinker by the addition of $1 \mathrm{M}$ Tris- $\mathrm{HCl}(\mathrm{pH} 7.4)$ to a final concentration of $20 \mathrm{mM}$ for $10 \mathrm{~min}$, cells were lysed for further analysis.

Immunofluorescence. Cells were fixed in $4 \%$ paraformaldehyde, incubated with anti-cytochrome $c$ (1:100; BD Biosciences), anti-Bax (1:100; Santa Cruz 
Biotechnology), anti-HA (1:200; Covance), anti-SOCS6 (1:50), anti-DRP1 (1:100; BD Biosciences), or anti-PGAM5 (1:100; Upstate Biotechnology) primary antibodies, and Alexa Flour 488-, 594-, or 350-conjugated secondary antibodies (Molecular Probes, Eugene, OR, USA) in a 1:200 dilution, counterstained with 4'6-diamidino-2-phenylindole (DAPI), and examined by fluorescence or confocal microscopy (LSM510 Meta, Carl Zeiss, Jena, Germany) with a $63 \times 1.4$ oil DIC Plan Apo objective or with a $100 \times 1.40$ NA oil immersion objective. For mitochondrial staining, $200 \mathrm{nM}$ MitoTracker Red CMXRos (Molecular Probes) was added to cultures $30 \mathrm{~min}$ before fixation.

Mitochondria isolation. Mitochondria were isolated using a mitochondria isolation kit (Thermo Scientific, Rockford, IL, USA) according to the manufacturer's instructions. Mitochondria were resuspended in isotonic buffer $(0.2 \mathrm{M}$ mannitol, $50 \mathrm{mM}$ sucrose, $1 \mathrm{mM}$ EDTA, $20 \mathrm{mM}$ HEPES-KOH, pH 7.4) or hypotonic buffer ( $5 \mathrm{mM}$ Tris, $\mathrm{pH} 7.4$, and $1 \mathrm{mM}$ EDTA), treated with $50 \mu \mathrm{g} / \mathrm{ml}$ trypsin for $30 \mathrm{~min}$ on ice, and analyzed by western blotting.

Mitochondrial morphology analysis (Micro-P). Cells were stained with MitoTracker and cell images were taken with a fluorescence microscope (BX51 equipped with a DP70 image acquisition system; Olympus, Tokyo, Japan). Cells were then analyzed by Micro-P software. ${ }^{27}$ Micro-P software measured individual mitochondrion according to the area, axial, and length/width, and sorted mitochondria into four types. ${ }^{27}$ Small fragmented mitochondria with an area $<20$ were first grouped as type 1. Next, the mitochondria with a length/width $<3$ were grouped into large fragmented mitochondria as type 2. Among the remaining mitochondria, the curved, branched, or circular tubular mitochondria with an axial $<2$ were grouped as type 3 , and the tubular mitochondrion with an axial $>2$ were designated type 4 .

Electron microscopy. Cells were fixed in $2.5 \%$ glutaraldehyde containing $0.1 \mathrm{M}$ phosphate buffer $(\mathrm{pH} 7.4)$ for $1 \mathrm{~h}$ and postfixed with $1 \%$ osmium in phosphate buffer for $90 \mathrm{~min}$. The dehydrated pellets were embedded into LR White acrylic resin. Thin sections (100 nm thick) were collected on $270-$ mesh nickel or gold grids. Samples were stained by floating on a fresh $50 \%$ aqueous saturated solution of uranyl acetate, rinsed twice with distilled water, and stained immediately with lead citrate. Thin sections were examined under a JEM1200EX electron microscope (JEOL, Tokyo, Japan). The lengths of CM and IBM length was measured using ImageJ software (NIH, Bethesda, MD, USA)

Cell fusion assay. For the cell fusion assay, cells individually labeled with mitochondria-targeted DsRed or EYFP were cocultured. Cells were then treated with $\mathrm{CHX}$ for $1 \mathrm{~h}$ and fused for $60 \mathrm{~s}$ with 50\% PEG1500 (Roche). After washing, cells were grown in $\mathrm{CHX}$-containing medium for the indicated times.

Fluorescence recovery after photobleaching. To measure FRAP, $1.5 \times 4.5-\mu \mathrm{m}$ regions of interest (ROIs) were photobleached by scanning the ROls with $561-n m$ laser line at full power $(100 \%)$. The entire cell was then scanned at 5 -ms intervals with low laser power (10\%) to monitor the recovery of fluorescence.

Measurement of $\Delta \psi \mathrm{m}$. Cells were harvested and $\Delta \psi \mathrm{m}$ was measured by staining cells with $1.5 \mu \mathrm{M} \mathrm{JC}-1$ (Molecular Probes) at $37^{\circ} \mathrm{C}$ for 30 min before flow cytometric analysis. The change in $\Delta \psi \mathrm{m}$ was expressed as FL2/FL1 using CellQuest software (BD Biosciences).

Cell metabolism assays. The cellular oxygen consumption rate and glycolysis rate were determined by measuring OCR and ECAR using a Seahorse XF24 Extracellular Flux Analyzer (Seahorse Bioscience, North Billerica, MA, USA). HeLa cells $\left(2 \times 10^{4}\right.$ cells/well) were adhered and incubated at $37^{\circ} \mathrm{C}$ for at least $1 \mathrm{~h}$ before OCR and ECAR analysis. Oligomycin, carbonyl cyanide p-trifluoromethoxyphenylhydrazone (FCCP), antimycin A, glucose, and 2-deoxyD-glucose (2-DG) were loaded into drug delivery ports and added sequentially. The ATP concentration in the cells was determined using an ATP Bioluminescence Assay kit (Roche) based on the light-emitting luciferase-catalyzed oxidation of luciferin. Lactate production was measured with a lactate reagent kit (Trinity Biotech, Bray, Ireland). The absorbance at $540 \mathrm{~nm}$ was measured by an enzyme-linked immunosorbent assay (ELISA) reader. For glucose uptake experiments, the uptake of the fluorescent glucose homolog 2-NBDG was measured by the addition of $20 \mathrm{nM}$ 2-NBDG in the medium for $2 \mathrm{~h}$ before flow cytometric analysis.
Statistical analyses. The results were expressed as the means \pm S.E.M. The $P$-values were two-tailed and performed using Student's $t$-test. Statistical significance was specified as $P<0.05$.

\section{Conflict of Interest}

The authors declare no conflict of interest.

Acknowledgements. This work was supported in part by National Science Council Grants (NSC95-2320-B-001-043-MY3 and NSC-101-2320-B-001-030MY3) to J-YC. We thank Dr. Ming-Jing Hwang and Ms. Ching-Shu Suen for assistance in bioinformatics.

1. Garrido C, Galluzzi L, Brunet M, Puig P, Didelot C, Kroemer G. Mechanisms of cytochrome C release from mitochondria. Cell Death Differ 2006; 13: 1423-1433.

2. Wang X. The expanding role of mitochondria in apoptosis. Genes Dev 2001; 15 : 2922-2933.

3. Cerveny KL, Tamura Y, Zhang Z, Jensen RE, Sesaki H. Regulation of mitochondrial fusion and division. Trends Cell Biol 2007; 17: 563-569.

4. Chan D. Mitochondria: dynamic organelles in disease, aging, and development. Cell 2006; 125: $1241-1252$.

5. Detmer SA, Chan DC. Functions and dysfunctions of mitochondrial dynamics. Nat Rev Mol Cell Biol 2007; 8: 870-879.

6. Grandemange S, Herzig S, Martinou JC. Mitochondrial dynamics and cancer. Semin Cancer Biol 2009; 19: 50-56.

7. Chen H, Chan DC. Mitochondrial dynamics-fusion, fission, movement, and mitophagy-in neurodegenerative diseases. Hum Mol Genet 2009; 18: R169-R176.

8. Praefcke G, McMahon $\mathrm{H}$. The dynamin superfamily: universal membrane tubulation and fission molecules? Nat Rev Mol Cell Biol 2004; 5: 133-147.

9. Chen H, Detmer S, Ewald A, Griffin E, Fraser S, Chan D. Mitofusins Mfn1 and Mfn2 coordinately regulate mitochondrial fusion and are essential for embryonic development. J Cell Biol 2003; 160: 189-200.

10. Olichon A, Baricault L, Gas N, Guillou E, Valette A, Belenguer P et al. Loss of OPA1 perturbates the mitochondrial inner membrane structure and integrity, leading to cytochrome c release and apoptosis. J Biol Chem 2003; 278: 7743-7746.

11. Smirnova E, Griparic L, Shurland DL, van der Bliek AM. Dynamin-related protein Drp1 is required for mitochondrial division in mammalian cells. Mol Biol Cell 2001; 12: 2245-2256.

12. Otera $\mathrm{H}$, Wang $\mathrm{C}$, Cleland MM, Setoguchi K, Yokota S, Youle RJ et al. Mff is an essentia factor for mitochondrial recruitment of Drp1 during mitochondrial fission in mammalian cells. J Cell Biol 2010; 191: 1141-1158.

13. James DI, Parone PA, Mattenberger Y, Martinou JC. hFis1, a novel component of the mammalian mitochondrial fission machinery. J Biol Chem 2003; 278: 36373-36379.

14. Wasilewski M, Scorrano L. The changing shape of mitochondrial apoptosis. Trends Endocrinol Metab 2009; 20: 287-294.

15. Chang $\mathrm{C}-\mathrm{R}$, Blackstone $\mathrm{C}$. Dynamic regulation of mitochondrial fission through modification of the dynamin-related protein Drp1. Ann NY Acad Sci 2010; 1201: 34-39.

16. Chang CR, Blackstone C. Cyclic AMP-dependent protein kinase phosphorylation of Drp1 regulates its GTPase activity and mitochondrial morphology. J Biol Chem 2007; 282 21583-21587.

17. Cereghetti G, Stangherlin A, De Brito OM, Chang C, Blackstone C, Bernardi P et al. Dephosphorylation by calcineurin regulates translocation of Drp1 to mitochondria. Proc Natl Acad Sci USA 2008; 105: 15803-15808.

18. Wang $Z$, Jiang $H$, Chen $S$, Du F, Wang $X$. The mitochondrial phosphatase PGAM5 functions at the convergence point of multiple necrotic death pathways. Cell 2012; 148: 228-243.

19. Jagasia R, Grote $P$, Westermann B, Conradt B. DRP-1-mediated mitochondrial fragmentation during EGL-1-induced cell death in C. elegans. Nature 2005; 433: 754-760.

20. Frank S, Gaume B, Bergmann-Leitner ES, Leitner WW, Robert EG, Catez F et al. The role of dynamin-related protein 1, a mediator of mitochondrial fission, in apoptosis. Dev Cell 2001; 1: 515-525.

21. Cassidy-Stone A, Chipuk JE, Ingerman E, Song C, Yoo C, Kuwana T et al. Chemical inhibition of the mitochondrial division dynamin reveals its role in Bax/Bak-dependent mitochondrial outer membrane permeabilization. Dev Cell 2008; 14: 193-204.

22. Cereghetti G, Costa V, Scorrano L. Inhibition of Drp1-dependent mitochondrial fragmentation and apoptosis by a polypeptide antagonist of calcineurin. Cell Death Differ 2010; 17: 1785-1794.

23. Lai RH, Hsiao YW, Wang MJ, Lin HY, Wu CW, Chi CW et al. SOCS6, down-regulated in gastric cancer, inhibits cell proliferation and colony formation. Cancer Lett 2010; 288: 75-85.

24. Masuhara M, Sakamoto $H$, Matsumoto A, Suzuki R, Yasukawa H, Mitsui K et al. Cloning and characterization of novel CIS family genes. Biochem Biophys Res Commun 1997; 239: 439-446. 
25. Nicholson SE, Willson TA, Farley A, Starr R, Zhang JG, Baca M et al. Mutational analyses of the SOCS proteins suggest a dual domain requirement but distinct mechanisms for inhibition of LIF and IL-6 signal transduction. EMBO J 1999; 18: 375-385.

26. Kim H, Tu HC, Ren D, Takeuchi O, Jeffers JR, Zambetti GP et al. Stepwise activation of BAX and BAK by tBID, BIM, and PUMA initiates mitochondrial apoptosis. Mol Cell 2009; 36: 487-499.

27. Peng JY, Lin CC, Chen YJ, Kao LS, Liu YC, Chou CC et al. Automatic morphological subtyping reveals new roles of caspases in mitochondrial dynamics. PLOS Comput Biol 2011; 7: e1002212.

28. Benard G, Bellance N, James D, Parrone P, Fernandez H, Letellier T et al. Mitochondrial bioenergetics and structural network organization. J Cell Sci 2007; 120: 838-848.

29. Smirnova E, Shurland D, Ryazantsev S, Van Der Bliek A. A human dynamin-related protein controls the distribution of mitochondria. J Cell Biol 1998; 143: 351-358.

30. Suen DF, Norris KL, Youle RJ. Mitochondrial dynamics and apoptosis. Genes Dev 2008; 22: $1577-1590$.

31. Karbowski M, Jeong SY, Youle RJ. Endophilin B1 is required for the maintenance of mitochondrial morphology. J Cell Biol 2004; 166: 1027-1039.

32. Oka T, Sayano T, Tamai S, Yokota S, Kato H, Fujii G et al. Identification of a novel protein MICS1 that is involved in maintenance of mitochondrial morphology and apoptotic release of cytochrome c. Mol Biol Cell 2008; 19: 2597-2608.

33. Krebs DL, Hilton DJ. A new role for SOCS in insulin action. Suppressor of cytokine signaling. Sci STKE 2003; 2003: PE6.

34. Kamura T, Maenaka K, Kotoshiba S, Matsumoto M, Kohda D, Conaway R et al. VHL-box and SOCS-box domains determine binding specificity for Cul2-Rbx1 and Cul5-Rbx2 modules of ubiquitin ligases. Genes Dev 2004; 18: 3055-3065.
35. Karbowski M, Youle RJ. Regulating mitochondrial outer membrane proteins by ubiquitination and proteasomal degradation. Curr Opin Cell Biol 2011; 23: 476-482.

36. Nakamura N, Kimura Y, Tokuda M, Honda S, Hirose S. MARCH-V is a novel mitofusin 2- and Drp1-binding protein able to change mitochondrial morphology. EMBO Rep 2006; 7: 1019-1022.

37. Wang $\mathrm{H}$, Song $\mathrm{P}, \mathrm{Du} \mathrm{L}$, Tian W, Yue W, Liu $\mathrm{M}$ et al. Parkin ubiquitinates Drp1 for proteasome-dependent degradation: implication of dysregulated mitochondrial dynamics in Parkinson disease. J Biol Chem 2011; 286: 11649-11658.

38. Zadjali F, Pike AC, Vesterlund M, Sun J, Wu C, Li SS et al. Structural basis for C-KIT inhibition by the suppressor of cytokine signaling 6 (SOCS6) ubiquitin ligase. J Biol Chem 2011; 286: 480-490.

39. Louis N, Evelegh C, Graham FL. Cloning and sequencing of the cellular-viral junctions from the human adenovirus type 5 transformed 293 cell line. Virology 1997; 233: 423-429.

40. Lai R, Wang M, Yang S, Chen J. Genomic organization and functional characterization of the promoter for the human suppressor of cytokine signaling 6 gene. Gene 2009; 448 : 64-73.

41. Lee JL, Wang MJ, Sudhir PR, Chen JY. CD44 engagement promotes matrix-derived survival through the CD44-SRC-integrin axis in lipid rafts. Mol Cell Biol 2008; 28: 5710-5723.

(c) This work is licensed under the Creative Commons Attribution-NonCommercial-No Derivative Works 3.0

Unported License. To view a copy of this license, visit http:// creativecommons.org/licenses/by-nc-nd/3.0/ 